\title{
ALGEBRAIC BIRKHOFF FACTORIZATION AND THE EULER-MACLAURIN FORMULA ON CONES
}

\author{
LI GUO, SYLVIE PAYCHA, AND BIN ZHANG
}

\begin{abstract}
AвstRact. We equip the space of lattice cones with a coproduct which makes it a connected cograded colagebra. The exponential sum and exponential integral on lattice cones can be viewed as linear maps on this space with values in the space of meromorphic germs with linear poles at zero. We investigate the subdivision properties- reminiscent of the inclusion-exclusion principle for the cardinal on finite sets- of such linear maps and establish a compatibility of these properties with respect to the convolution quotient of the coalgebra. Implementing the Algebraic Birkhoff Factorization procedure on the linear maps under consideration, we factorize the exponential sum as a convolution quotient of two maps, with each of the maps in the factorization satisfying a subdivision property. Consequently, the Algebraic Birkhoff Factorization specializes to the EulerMaclaurin formula on lattice cones and provides a simple formula for the interpolating factor by means of a projection map.
\end{abstract}

\section{Contents}

1. Introduction

2. Lattice cones and their coproduct

2.1. Lattice cones

2.2. Transverse lattice cones

2.3. The coalgebra of lattice cones

3. Subdivision properties

3.1. Subdivisions of lattice cones

3.2. Induced subdivisions on transverse cones

3.3. Compatibility of the convolution product with subdivisions

4. Euler-Maclaurin formulae for lattice cones

4.1. Meromorphicity of generating functions 22

4.2. Algebraic Birkhoff Factorization

4.3. Euler-Maclaurin formula 28

References

\section{INTRODUCTION}

The classical Euler-Maclaurin formula in analysis [12] and its higher dimensional generalizations [ㄴ, 4, 17] express Riemann sums in terms of integrals over polytopes. Their geometric relevance in relation with the Riemann-Roch theorem on toric varieties arises from the appearance of the Todd operators [8], related to the Todd classes of the toric varieties associated with the polytopes. We study these formulae applying Algebraic Birkhoff Factorization from a renormalization method in quantum field theory.

Date: October 29, 2018.

2010 Mathematics Subject Classification. 11H06, 52C07, 52B20, 65B15, $11 \mathrm{M} 32$.

Key words and phrases. convex cones, coalgebras, Algebraic Birkhoff Factorization, Euler-Maclaurin formula, meromorphic functions, subdivision property . 
The idea of this approach comes from two observations. In [3] localized formulae for equivariant Todd classes of toric varieties are given, which make explicit the geometric nature of the localized formulae. A natural question is how to recover the equivariant or ordinary Todd classes from the localized formulae. From a mathematical viewpoint, this amounts to extracting them from fractions arising in the localized formula, and from the viewpoint of physics, it boils down to dealing with the singularities, an issue which calls for a renormalization procedure. On the other hand, the exponential sum on a cone can be viewed as a regularization of the ill-defined partition function $\sum_{\vec{n} \in C \cap \mathbb{Z}^{k}} 1$ over a cone $C$ in $\mathbb{R}^{k}$. This suggests the application of a renormalization process.

Precisely, on a convex polyhedral convex cone, the exponential sum in Eq. (37) and exponential integral in Eq. (35) can be viewed as morphisms with values in the space of multivariate meromorphic germs with linear poles at zero. We interpolate the exponential sum and exponential integral by means of an Algebraic Birkhoff Factorization implemented on geometric cones, inspired by the algebraic renormalization scheme of Connes and Kreimer. Let us briefly recall their approach.

Theorem (Algebraic Birkhoff Factorization)[5] Let $H$ be a commutative connected filtered Hopf algebra. Let $R$ be a commutative algebra with a Rota-Baxter operator $P$ of weight -1 . Let $\phi: H \rightarrow R$ be an algebra homomorphism.

(a) There are algebra homomorphisms $\phi_{-}: H \rightarrow \mathbf{k}+P(R)$ and $\phi_{+}: H \rightarrow \mathbf{k}+(\mathrm{id}-P)(R)$, with $\mathbf{k}$ being the base ring, such that

$$
\phi=\phi_{-}^{*(-1)} * \phi_{+}
$$

Here $\phi_{-}^{*(-1)}$ is the inverse of $\phi_{-}$with respect to the convolution product $*$ on the space of linear maps from $H$ to $R$ associated with the coproduct on $H$.

(b) If $P^{2}=P$, then the decomposition in (a) is unique.

In our context, the projection $P$ does not satisfy the Rota-Baxter property, so we first need to generalize Connes and Kreimer's approach. By identifying the factors in the Algebraic Birkhoff Factorization, we then show how the Algebraic Birkhoff Factorization indeed gives the EulerMaclaurin formula. This approach has the extra benefit of providing a simple formula for the interpolation function.

As the context to apply the Algebraic Birkhoff Factorization, we introduce the notion of lattice cones (Definition 2.9), which are pairs consisting of a cone and a lattice, needed to make sense of exponential generating sums relative to a choice of lattice points. On lattice cones, the exponential generating sum $S^{c}$ in Eq. (37) and exponential integral $I$ in Eq. (35), first defined on simplicial lattice cones and then extended to general lattice cones by subdivisions, yield meromorphic germs with linear poles. Thus, the linear extensions to the linear space generated by lattice cones give linear maps with values in the space of meromorphic germs with linear poles at zero.

To construct the coproduct in the space of lattice cones needed for implementing the Algebraic Birkhoff Factorization, we fix an inner product (see Eq. (2)) on the underlying space of the lattice cones. Borrowing the definition of transverse cone from [1], defined by means of this inner product, we build the coproduct in Eq. (8) on the space of lattice cones from a complement map which assigns to a face of a lattice cone the transverse lattice cone (Proposition 2.22). This coproduct is compatible with the partial order and the dimension filtration on cones; Theorem 2.25 endows 
the space of lattice cones with a connected cograded coalgebra structure. The corresponding convolution product (Lemma 4.9) on the algebra of linear maps from the space of lattice cones to a commutative algebra is later used for the Algebraic Birkhoff Factorization.

The Algebraic Birkhoff Factorization in the renormalization scheme of Connes and Kreimer requires the regularized linear map to take values in a Rota-Baxter algebra. The fact that the range of our linear maps being the space of meromorphic germs with linear poles at zero imposes a special treatment. This is one of our motivations to investigate the structure of the space of meromorphic germs with linear poles at zero [11]. It turns out that this space is a commutative algebra which splits into a subalgebra and a complement of it which is not a subalgebra. Consequently, the projection is not a Rota-Baxter operator, and the decomposition depends on a choice of an inner product. Theorem 4.10 which in contrast does apply to the present situation, generalizes the Algebraic Birkhoff Factorization to linear maps on a connected cograded coalgebra which is not necessarily a Hopf algebra, with values in a commutative algebra which splits into a subalgebra and its complement.

Having the necessary ingredients at hand, we then apply the (generalized) Algebraic Birkhoff Factorization to the exponential generating sum, and obtain a factorization in terms of a "holomorphic" part and a "polar part" (Corollary 4.11).

Our next step is to derive the Euler-Maclaurin formula as a special case of the Algebraic Birkhoff Factorization, when the inner product used to define the transverse cone is assumed to coincide with the inner product to define the projection $\pi_{+}$(in Eq. (42) ) onto the holomorphic part of the space of meromorphic germs with linear poles at zero. For this purpose, we only need to identity the "polar part" of the Algebraic Birkhoff Factorization with the exponential integral in the Euler-Maclaurin formula, which is clear for smooth cones. In order to apply it to general lattice cones by means of subdivisions, we carry out a detailed study of the different types of subdivision properties (Definition 3.11) enjoyed by the exponential generating sum and exponential integral, including closed discrete type for the sum and of continuous type for the integral. This is reminiscent of the inclusion-exclusion principle in set theory and the sieve method in number theory.

The compatibility of subdivision properties of the factors with the convolution quotient in the Algebraic Birkhoff Factorization is investigated in the general result Theorem 3.13. It states that the convolution quotient of two maps on the coalgebra of lattice cones with values in a commutative algebra, both of which satisfy the discrete closed subdivision property, satisfies the continuous subdivision property.

Returning to our case of the exponential generating sum and exponential integral, the fact (Theorem 4.13) that the "holomorphic part" coincides with the holomorphic projection of the exponential generating sum, implies that it satisfies the discrete closed subdivision property (see Corollary 4.14.(国)). Theorem 3.13 applied to the Algebraic Birkhoff Factorization of the exponential generating sum, leads to Corollary 4.14.(伍) which states that the "polar part" satisfies the continuous subdivision property. Based on the fact which results from a straightforward calculation, that for smooth lattice cones, the "polar part" is the exponential integral, the compatibility with subdivisions yields that the "polar part" coincides with the exponential integral for general lattice cones. Consequently, the Algebraic Birkhoff Factorization amounts to the the Euler-Maclaurin formula. 


\section{LATTICE CONES AND THEIR COPRODUCT}

In this section, we introduce the concepts of lattice cones and a transverse lattice cones to faces of lattice cones. Using transverse lattice cones, we equip the linear span of lattice cones with a coalgebra structure.

2.1. Lattice cones. In a finite dimensional vector space over $\mathbb{R}$, a lattice is a finitely generated subgroup which spans the whole space. A real vector space equipped with a lattice is called a lattice space A rational multiple of a vector in the lattice is called a rational lattice vector.

Definition 2.1. A filtered lattice space is a pair $(V, \Lambda)$ from a family $\left(V_{k}, \Lambda_{k}\right), k \geq 1$, of lattice vector spaces such that $V_{1} \subset V_{2} \subset \cdots, V=\cup_{k=1}^{\infty} V_{k}, \Lambda_{k}=\Lambda_{k+1} \cap V_{k}$ and $\Lambda=\cup_{k=1}^{\infty} \Lambda_{k}$.

Remark 2.2. In applications, the filtered lattice space usually is $\mathbb{R}^{\infty}$ with $V_{k}=\mathbb{R}^{k}, \Lambda_{k}$ the standard lattice $\mathbb{Z}^{k}$, and $\left\{e_{1}, e_{2}, \cdots\right\}$ the canonical basis.

We now collect basic definitions and facts (mostly following [6] and [18]) on cones that will be used in this paper. See [10] for a detailed discussion on these facts. For a subset $S$ of $V$, let $\operatorname{lin}(S)$ denote its $\mathbb{R}$-linear span.

(a) By a cone in $V_{k}$ we mean a closed convex polyhedral cone in $V_{k}$, namely the convex set

$$
\left\langle v_{1}, \cdots, v_{n}\right\rangle:=\mathbb{R}\left\{v_{1}, \cdots, v_{n}\right\}=\mathbb{R}_{\geq 0} v_{1}+\cdots+\mathbb{R}_{\geq 0} v_{n},
$$

where $v_{i} \in \Lambda_{k}, i=1, \cdots, n$.

(b) The set $\left\{v_{1}, \cdots, v_{n}\right\}$ in Eq. (ID) is called a generating set or a spanning set of the cone.

(c) The spanning set $\left\{v_{1}, \cdots, v_{n}\right\}$ is called primary if

(i) $v_{i} \in \Lambda_{k}, i=1, \cdots, n$,

(ii) there is no real number $r_{i} \in(0,1)$ such that $r_{i} v_{i}$ lies in $\Lambda_{k}$, and

(iii) none of the generating vectors $v_{i}$ is a positive linear combination of the others.

For a lattice cone, its primary generating set exists.

(d) Define the dimension of a cone $C$ by $\operatorname{dim} C:=\operatorname{dim} \operatorname{lin}(C)$.

(e) A cone is called strongly convex if it does not contain any nonzero linear subspace.

(f) A simplicial cone is a cone spanned by linearly independent vectors. A simplicial cone is strongly convex.

(g) A smooth cone is a cone whose primary generating set is a part of a lattice basis of $\Lambda_{k} \subseteq V_{k}$. For a full dimensional cone, smoothness is equivalent to the unimodularity, namely that the determinant of the transformation matrix relating the primary generating set to a basis of $\Lambda_{k}$ is \pm 1 .

(h) A face of a cone $C$ is a subset of the form $C \cap\{u=0\}$, where $u: V_{k} \rightarrow \mathbb{R}$ is a linear form that is non-negative on $C$. A face $F$ of a cone $C$ is again a cone and we write $F \leq C$. If $F$ is a proper face of a cone $C$ we write $F \supsetneqq C$.

Example 2.3. A Chen cone $C_{k}^{\text {Chen }}$, defined by $\left\langle e_{1}, e_{1}+e_{2}, \cdots, e_{1}+\cdots+e_{k}\right\rangle$, is a smooth cone.

Lemma 2.4. Let $W \subset U$ be subspaces of lattice space $\left(V_{k}, \Lambda_{k}\right)$ spanned by lattice vectors and let $\Lambda_{U}$ be a lattice of $U$ with lattice vectors. Then $W \cap \Lambda_{U}$ is a lattice of $W$.

Proof. Let $\left\{w_{1}, \cdots, w_{m}\right\}$ be a basis of $W$ with lattice vectors and $\left\{u_{1}, \cdots, u_{\ell}\right\}$ a basis of $\Lambda_{U}$ with lattice vectors. Then for $i=1, \cdots m, w_{i}$ is a rational combination of $u_{1}, \cdots, u_{\ell}$. Therefore there exist $0 \neq r_{i} \in \mathbb{Z}$ such that $r_{i} w_{i} \in \Lambda_{U}, i=1, \cdots, m$. Then we have $W=\sum_{i=1}^{m} \mathbb{R} r_{i} w_{i} \subseteq \mathbb{R}\left(W \cap \Lambda_{U}\right)$. Since $W \cap \Lambda_{U}$ is also finitely generated, it is a lattice of $W$. 
On the grounds of Lemma 2.4, we set the following definition.

Definition 2.5. (a) A lattice cone in $V_{k}$ is a pair $\left(C, \Lambda_{C}\right)$ with $C$ a cone in $V_{k}$ and $\Lambda_{C}$ a lattice in $\operatorname{lin}(C)$ generated by lattice vectors.

(b) A face of a lattice cone $\left(C, \Lambda_{C}\right)$ is the lattice cone $\left(F, \Lambda_{F}\right)$ where $F$ is a face of $C$ and $\Lambda_{F}:=\Lambda_{C} \cap \operatorname{lin}(F)$.

(c) A primary generating set of a lattice cone $\left(C, \Lambda_{C}\right)$ is a generating set $\left\{v_{1}, \cdots, v_{n}\right\}$ of $C$ such that

(i) $v_{i} \in \Lambda_{C}, i=1, \cdots, n$,

(ii) there is no real number $r_{i} \in(0,1)$ such that $r_{i} v_{i}$ lies in $\Lambda_{C}$, and

(iii) none of the generating vectors $v_{i}$ is a positive linear combination of the others.

Remark 2.6. (a) Any lattice cone possesses a primary generating set: starting from any lattice generating set $\left\{v_{1}, \cdots, v_{n}\right\}$ of the lattice cone, a rescaling yields a set satisfying the first two conditions. A primary generating set is obtained by eliminating an element if it is a combination of the remaining ones.

(b) For a strongly convex lattice cone, a primary generating set is unique: it consists of the shortest lattice vector in each of the spanning vectors of cone [6].

(c) For a cone $C \subseteq V_{k}$, the primary generating set of the lattice cone $\left(C, \operatorname{lin}(C) \cap \Lambda_{k}\right)$ coincides with that of the cone $C$.

The following properties of lattice cones are easy to verify.

Lemma 2.7. Let $\left(C, \Lambda_{C}\right)$ be a lattice cone.

(a) Let $C^{\prime}$ be a lattice cone with $\operatorname{lin}(C)=\operatorname{lin}\left(C^{\prime}\right)$. Then $\left(C^{\prime}, \Lambda_{C}\right)$ is also a lattice cone.

(b) If $F$ is the face of another face $G$ of $C$, then $\Lambda_{F}=\Lambda_{G} \cap \operatorname{lin}(F)$.

Example 2.8. The lattice cone $\left(\left\langle e_{1}\right\rangle, \mathbb{Z} e_{1}\right)$ is a face of the lattice cone $\left(\left\langle e_{1}, e_{2}\right\rangle, \mathbb{Z} e_{1}+\mathbb{Z} e_{2}\right)$. It is also a face of the lattice cone $\left(\left\langle e_{1}, e_{2}\right\rangle, \mathbb{Z}\left(e_{1}+e_{2}\right)+\mathbb{Z} e_{2}\right)$ since $r e_{1} \in \mathbb{Z}\left(e_{1}+e_{2}\right)+\mathbb{Z} e_{2}$ if and only if $r \in \mathbb{Z}$. But it is not a face of the lattice cone $\left(\left\langle e_{1}, e_{2}\right\rangle, \mathbb{Z}\left(e_{1}+e_{2}\right)+\mathbb{Z}\left(e_{1}-e_{2}\right)\right)$ since $r e_{1} \in \mathbb{Z}\left(e_{1}+e_{2}\right)+\mathbb{Z}\left(e_{1}-e_{2}\right)$ if and only if $r \in 2 \mathbb{Z}$. This also shows that $\left(\left\langle e_{1}\right\rangle, \mathbb{Z} 2 e_{1}\right)$ is a face of the lattice cone $\left(\left\langle e_{1}, e_{2}\right\rangle, \mathbb{Z}\left(e_{1}+e_{2}\right)+\mathbb{Z}\left(e_{1}-e_{2}\right)\right)$.

Definition 2.9. A lattice cone $\left(C, \Lambda_{C}\right)$ is called strongly convex (resp. simplicial) if $C$ is. A lattice cone $\left(C, \Lambda_{C}\right)$ is called smooth if the additive monoid $\Lambda_{C} \cap C$ has a monoid basis. In other words, $\left(C, \Lambda_{C}\right)$ is called smooth if and only if there are linearly independent lattice vectors $v_{1}, \cdots, v_{\ell}$ such that $\Lambda_{C} \cap C=\mathbb{Z}_{\geq 0}\left\{v_{1}, \cdots, v_{\ell}\right\}$.

The following facts are easy to check.

Remark 2.10. (a) For any simplicial cone $C$ spanned by linearly independent lattice vectors $v_{1}, \cdots, v_{n}$, the lattice cone $\left(C, \mathbb{Z}\left\{v_{1}, \cdots, v_{n}\right\}\right)$ is smooth;

(b) The smoothness of a cone comapre with that of a lattice cone, for a cone $C$ in $V_{k}$ is smooth if and only if the lattice cone $\left(C, \Lambda_{k} \cap \operatorname{lin}(C)\right)$ is smooth.

Example 2.11. The lattice cone $\left(\left\langle e_{1}, e_{2}\right\rangle, \mathbb{Z} e_{1}+\mathbb{Z} e_{2}\right)$ is smooth. By the first remark, the lattice cone $\left(\left\langle e_{1}, e_{1}+2 e_{2}\right\rangle, \mathbb{Z} e_{1}+\mathbb{Z} 2 e_{2}\right)$ is smooth even though $\left\langle e_{1}, e_{1}+2 e_{2}\right\rangle$ is not smooth. By the second remark the lattice cone $\left(\left\langle e_{1}, e_{1}+2 e_{2}\right\rangle, \mathbb{Z} e_{1}+\mathbb{Z} e_{2}\right)$ is not smooth.

\footnotetext{
${ }^{1}$ The relevance of a chosen lattice in a vector space is mentioned in [1] (see the word of caution in par. 4). The term lattice cone can also be found in the literature on Banach spaces with a somewhat different meaning [14].
} 
The following elementary property is useful for later purposes.

Proposition 2.12. A face of a smooth lattice cone $\left(C, \Lambda_{C}\right)$ is smooth.

Proof. Let $\left(F, \Lambda_{F}\right)$ be a face of a smooth lattice cone $\left(C, \Lambda_{C}\right)$. Let $u: V_{k} \rightarrow \mathbb{R}$ be a linear function defining the face $F:=C \cap u^{\perp}$. Then $\Lambda_{F}=\Lambda_{C} \cap \operatorname{lin}(F)$. Let $\left\{v_{1}, \cdots, v_{m}\right\}$ be a monoid basis of $C \cap \Lambda_{C}$. To prove that $\left(F, \Lambda_{F}\right)$ is smooth, we only need to show that the set $\left\{v_{1}, \cdots, v_{m}\right\} \cap u^{\perp}$ is a monoid basis of $\Lambda_{F} \cap F$. Since the set is linearly independent, this amounts to showing that it generates $\Lambda_{F} \cap F$ as a monoid.

Let $v \in \Lambda_{F} \cap F$. Note that $\Lambda_{F} \cap F=\Lambda_{C} \cap \operatorname{lin}(F) \cap C \cap u^{\perp}=\Lambda_{C} \cap C \cap u^{\perp}$. Thus for $v \in \Lambda_{F} \cap F$, from $v \in \Lambda_{C}$ we have $v=\sum_{i=1}^{m} a_{i} v_{i}$ with $a_{i} \in \mathbb{Z}$. From $v \in C$ we also have $a_{i} \in \mathbb{Z}_{\geq 0}$. For $v \in u^{\perp}$ we further have $0=u(v)=\sum_{i=1}^{m} a_{i} u\left(v_{i}\right)$. Thus if $a_{i} \neq 0$, then $u\left(v_{i}\right)=0$. It follows that $v \in \sum_{i, u\left(v_{i}\right)=0} \mathbb{Z}_{\geq 0} v_{i}=\mathbb{Z}_{\geq 0}\left\{\left\{v_{1}, \cdots, v_{m}\right\} \cap u^{\perp}\right\}$, which completes the proof.

2.2. Transverse lattice cones. Let $\mathfrak{C}_{k}$ denote the set of lattice cones in $V_{k}, k \geq 1$. The natural inclusions $\mathfrak{C}_{k} \rightarrow \mathfrak{C}_{k+1}$ induced by the natural inclusions $V_{k} \rightarrow V_{k+1}, \Lambda_{k} \rightarrow \Lambda_{k+1}, k \geq 1$, give rise to the direct limit $\mathfrak{C}=\lim _{\longrightarrow} \mathfrak{C}_{k}=\cup_{k \geq 1} \mathfrak{C}_{k}$.

We want to equip the $\mathbb{Q}$-linear space $\mathbb{Q} \mathfrak{C}$ generated by $\mathfrak{C}$ with a coproduct by applying the concept of a transverse cone borrowed from [1] and enriched to lattice cones.

We use an inner product on a filtered lattice space to identify quotient spaces and subspaces. This can be done by means of more general complement maps as in [7] but, in this paper, we choose to use the inner product for that purpose.

Definition 2.13. Let $V:=\cup_{k \geq 1} V_{k}$ with $\Lambda=\cup_{k \geq 1} \Lambda_{k}$ be a filtered lattice space. An inner product $Q(\cdot, \cdot)=(\cdot, \cdot)$ on $V$ is a sequence of inner products

$$
Q_{k}(\cdot, \cdot)=(\cdot, \cdot)_{k}: V_{k} \otimes V_{k} \rightarrow \mathbb{R}, \quad k \geq 1,
$$

that is compatible with the inclusion $j_{k}: V_{k} \hookrightarrow V_{k+1}$ and whose restriction to $\Lambda \otimes \mathbb{Q}$ and hence $\Lambda$ takes values in $\mathbb{Q}$. A filtered lattice vector space together with an inner product on $V$ is called a filtered lattice Euclidean space.

From now on, our discussion is on a fixed filtered lattice Euclidean space $(V, \Lambda)$ with the Euclidean inner product

$$
Q(\cdot, \cdot)=(\cdot, \cdot)
$$

and we drop $Q$ from the superscript to simplify notations whenever there is no ambiguity. Let $L$ be a lattice subspace of $V_{k}$. Set

$$
L^{\perp_{k}}:=L^{\perp_{k}^{Q}}:=\left\{v \in V_{k} \mid Q_{k}(v, u)=0 \text { for all } u \in L\right\} .
$$

The inner product $Q_{k}$ gives the direct sum decomposition $V_{k}=L \oplus L^{\perp_{k}}$ and hence the orthogonal projection

$$
\pi_{k, L^{\perp}}:=\pi_{k, L^{\perp}}^{Q}: V_{k} \rightarrow L^{\perp_{k}}
$$

along $L$. Also, the induced isomorphism $Q_{k}^{*}: V_{k} \rightarrow V_{k}^{*}$ yields an embedding $V_{k}^{*} \hookrightarrow V_{k+1}^{*}$. We refer to the direct limit $V^{\circledast}:=\bigcup_{k=1}^{\infty} V_{k}^{*}=\lim _{\longrightarrow} V_{k}^{*}$ as the filtered dual space of $V$. In general $V^{\circledast}$ differs from the usual dual space $V^{*}$. 
Example 2.14. Let $V=\mathbb{R}^{\infty}$ be equipped with the canonical inner product. For $L=\operatorname{lin}\left(e_{1}+e_{2}\right) \subset$ $V_{2}=\mathbb{R}^{2}$. We have $V_{2} / L \simeq L^{\perp_{2}}=\operatorname{lin}\left(e_{1}-e_{2}\right)$.

Definition 2.15. ([1]]) Let $F$ be a face of a cone $C \subseteq V_{k}$. The transverse cone $t(C, F)$ to $F$ is the projection $\pi_{k, F^{\perp}}(C)$ of $C$ in $\operatorname{lin}(F)^{\perp} \subseteq V_{k}$, where $\pi_{k, F^{\perp}}=\pi_{k, \operatorname{lin}(F)^{\perp}}$.

Note that $t(C, F)$ might not be a face of $C$. For example, the transverse cone to the face $F=$ $\left\langle e_{1}+e_{2}\right\rangle$ of the cone $C=\left\langle e_{1}, e_{1}+e_{2}\right\rangle$ is the cone $t(C, F)=\left\langle e_{1}-e_{2}\right\rangle$ under the standard inner product.

The commutative diagram

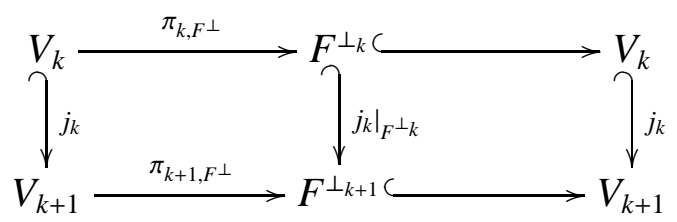

shows that $\pi_{k, F^{\perp}}(C)$ is actually independent of the choice of $k \geq 1$ such that $C \subseteq V_{k}$. Thus $t(C, F)$ is well-defined in $\mathfrak{C}$. So we can simplify the above notations $\pi_{k, L^{\perp}}$ by dropping the subscript $k$.

Lemma 2.16. For a face $F$ of $C$, the transverse cone $t(C, F)$ is strongly convex.

Proof. Assume that the face $F$ is given by a linear functional $u$, i.e., $F=C \cap\{u=0\}$. If the transverse cone $t(C, F)$ is not strongly convex, then there is a nonzero vector $v \in t(C, F)$, such that $-v \in t(C, F)$. By the definition of transverse cone, there are vectors $v^{\prime} \in \operatorname{lin}(F)$ and $v^{\prime \prime} \in \operatorname{lin}(F)$ such that $v+v^{\prime} \in C$ and $-v+v^{\prime \prime} \in C$. Since $v$ is nonzero, we have $v+v^{\prime} \notin F$, so $u\left(v+v^{\prime}\right)=u(v)>0$. For the same reason, we have $u(-v)=u\left(-v+v^{\prime \prime}\right)>0$, which is a contradiction.

We next generalize the concept of transverse cones to the context of lattice cones. Let $\left(C, \Lambda_{C}\right)$ be a lattice cone in $V_{k}$. Under the projection $\pi_{F^{\perp}}: V_{k} \rightarrow \operatorname{lin}(F)^{\perp}$, the lattice cone $C$ is sent to a lattice cone. Also the lattice $\Lambda_{C}$ in $\operatorname{lin}(C)$ is sent to a lattice in $\pi_{F^{\perp}}(\operatorname{lin}(C))$ since $\pi_{F^{\perp}}\left(\Lambda_{C}\right)$ is a finitely generated abelian group and $\operatorname{spans} \operatorname{lin}(t(C, F))=\pi_{F^{\perp}}(\operatorname{lin}(C))$. This justifies the following definition.

Definition 2.17. Let $\left(F, \Lambda_{F}\right)$ be a face of the lattice cone $\left(C, \Lambda_{C}\right)$ in $V_{k}$. The transverse lattice cone $\left(t(C, F), \Lambda_{t(C, F)}\right)$ along the face $\left(F, \Lambda_{F}\right)$ is the image of $\left(C, \Lambda_{C}\right)$ under the projection $\pi_{F^{\perp}}$ :

$$
\left(t(C, F), \Lambda_{t(C, F)}\right):=\left(\pi_{F^{\perp}}(C), \pi_{F^{\perp}}\left(\Lambda_{C}\right)\right) .
$$

We also use the notation $t\left(\left(C, \Lambda_{C}\right),\left(F, \Lambda_{F}\right)\right)$ to denote the transverse lattice cone.

Remark 2.18. In general, $\Lambda_{t(C, F)} \neq \Lambda_{C} \cap \operatorname{lin}(t(C, F))$, see the example below and the word of caution in par. 4 of [四].

Example 2.19. Using the standard inner product and the induced lattice, the transverse lattice cone to the face $\left(F, \Lambda_{F}\right)=\left(\left\langle e_{1}+e_{2}\right\rangle, \Lambda_{2} \cap \operatorname{lin}\left(e_{1}+e_{2}\right)\right)$ of the cone $\left(C, \Lambda_{C}\right)=\left(\left\langle e_{1}, e_{1}+e_{2}\right\rangle, \Lambda_{2}\right)$ is $\left(t(C, F), \Lambda_{t(C, F)}\right)=\left(\left\langle e_{1}-e_{2}\right\rangle, \mathbb{Z}\left(\frac{e_{1}-e_{2}}{2}\right)\right)$, so that $\Lambda_{t(C, F)} \neq \Lambda_{2} \cap \operatorname{lin}\left(e_{1}-e_{2}\right)=\mathbb{Z}\left(e_{1}-e_{2}\right)$.

For faces $F \leq G \leq C$ of the cone $C$, the transverse cone $t(G, F)$ can be viewed as a face of $t(C, F)$ and as the transverse cone $\pi_{F^{\perp}}(G)$. Thus the lattice $\Lambda_{t(G, F)}$ of $t(G, F)$ can be defined in two ways, firstly as the lattice of the face $t(G, F)$ of $t(C, F)$, namely $\Lambda_{t(G, F)}:=\Lambda_{t(C, F)} \cap \operatorname{lin}(t(G, F))$, and alternatively as the lattice of the transverse cone $t(G, F)$, namely $\Lambda_{t(G, F)}:=\pi_{F^{\perp}}\left(\Lambda_{G}\right)$. We need to verify that the two definitions agree. For this we first prove a lemma.

Lemma 2.20. We have $\pi_{F^{\perp}}\left(\Lambda_{C} \cap \operatorname{lin}(G)\right)=\pi_{F^{\perp}}\left(\Lambda_{C}\right) \cap \pi_{F^{\perp}}(\operatorname{lin}(G))$. 
Proof. The left hand side is clearly contained in the right hand side. On the other hand, for $w \in \pi_{F^{\perp}}\left(\Lambda_{C}\right) \cap \pi_{F^{\perp}}(\operatorname{lin}(G))$, there are $x \in \Lambda_{C}$ and $y \in \operatorname{lin}(G)$ such that $w=\pi_{F^{\perp}}(x)=\pi_{F^{\perp}}(y)$. So $\pi_{F^{\perp}}(x-y)=0$, that is, $x-y \in \operatorname{lin}(F) \subset \operatorname{lin}(G)$, implying $x \in \operatorname{lin}(G)$. Thus $x$ is in $\Lambda_{C} \cap \operatorname{lin}(G)$ and $w$ is contained in the left hand side.

The following proposition shows the equivalence of the two definitions of $\Lambda_{t(G, F)}$.

Proposition 2.21. For $F \leq G \leq C$, we have

$$
\Lambda_{t(G, F)}:=\Lambda_{t(C, F)} \cap \operatorname{lin}(t(G, F))=\pi_{F^{\perp}}\left(\Lambda_{G}\right) .
$$

Proof. Applying Lemma 2.20, we obtain

$$
\begin{aligned}
\pi_{F^{\perp}}\left(\Lambda_{G}\right) & =\pi_{F^{\perp}}\left(\Lambda_{C} \cap \operatorname{lin}(G)\right) \\
& =\pi_{F^{\perp}}\left(\Lambda_{C}\right) \cap \pi_{F^{\perp}}(\operatorname{lin}(G)) \\
& =\pi_{F^{\perp}}\left(\Lambda_{C}\right) \cap \operatorname{lin}\left(\pi_{F^{\perp}}(G)\right) \\
& =\Lambda_{t(C, F)} \cap \operatorname{lin}(t(G, F)) .
\end{aligned}
$$

Thus the two definitions of $\Lambda_{t(G, F)}$ agree.

Proposition 2.22. Transverse cones enjoy the following properties. Let $F$ be a face of a cone $C$.

(a) (Transitivity) $t(C, F)=t\left(t\left(C, F^{\prime}\right), t\left(F, F^{\prime}\right)\right)$ if $F^{\prime}$ is a face of $F$.

(b) (Compatibility with the partial order) We have $\{H \leq t(C, F)\}=\{t(G, F) \mid F \leq G \leq C\}$.

(c) (Compatibility with the dimension filtration) $\operatorname{dim}(C)=\operatorname{dim}(F)+\operatorname{dim}(t(C, F)$ ) for any face $F$ of $C$.

To the first two properties correspond similar properties for lattice cones.

(d) (Transitivity) $t\left(\left(C, \Lambda_{C}\right),\left(F, \Lambda_{F}\right)\right)=t\left(t\left(\left(C, \Lambda_{C}\right),\left(F^{\prime}, \Lambda_{F^{\prime}}\right)\right), t\left(\left(F, \Lambda_{F}\right),\left(F^{\prime}, \Lambda_{F^{\prime}}\right)\right)\right)$ if $\left(F^{\prime}, \Lambda_{F^{\prime}}\right)$ is a face of $\left(F, \Lambda_{F}\right)$.

(e) (Compatibility with the partial order) We have

$$
\left\{\left(H, \Lambda_{H}\right) \leq t\left(\left(C, \Lambda_{C}\right),\left(F, \Lambda_{F}\right)\right)\right\}=\left\{\left(t\left(\left(G, \Lambda_{G}\right),\left(F, \Lambda_{F}\right)\right) \mid\left(F, \Lambda_{F}\right) \leq\left(G, \Lambda_{G}\right) \leq\left(C, \Lambda_{C}\right)\right\} .\right.
$$

Proof. We first carry out the proof for ordinary cones.

(a) For $F^{\prime} \leq F \leq C$, we have $\operatorname{lin}\left(F^{\prime}\right)<\operatorname{lin}(F)<V_{k}$. Thus the inner product induces orthogonal decompositions

$$
V_{k}=\operatorname{lin}(F) \oplus \operatorname{lin}(F)^{\perp}, \quad \operatorname{lin}(F)=\operatorname{lin}\left(F^{\prime}\right) \oplus L .
$$

Therefore

$$
V_{k}=\operatorname{lin}\left(F^{\prime}\right) \oplus L \oplus \operatorname{lin}(F)^{\perp}, \quad \operatorname{lin}\left(F^{\prime}\right)^{\perp}=L \oplus \operatorname{lin}(F)^{\perp} .
$$

By definition, we have

$$
L=\operatorname{lin}\left(t\left(F, F^{\prime}\right)\right), \quad L^{\perp}=\operatorname{lin}\left(F^{\prime}\right) \oplus \operatorname{lin}(F)^{\perp} .
$$

This implies $\pi_{F^{\perp}}=\pi_{t\left(F, F^{\prime}\right)^{\perp}} \pi_{F^{\prime}}$. \. Thus we have

$$
t(C, F)=\pi_{F^{\perp}}(C)=\left(\pi_{t\left(F, F^{\prime}\right)^{\perp}} \pi_{F^{\prime}}\right)(C)=\pi_{t\left(F, F^{\prime}\right)^{\perp}}\left(t\left(C, F^{\prime}\right)\right)=t\left(t\left(C, F^{\prime}\right), t\left(F, F^{\prime}\right)\right) .
$$

(b) Assume that $F$ is defined by a linear form $u_{F} \in V^{*}$. Let $G$ be a face of $C$ containing $F$ that is defined by $u_{G} \in V^{*}$. Then $\left.u_{G}\right|_{F}=0$. But any element $u \in V^{*}$ with $\left.u\right|_{F}=0$ induces an element

\footnotetext{
${ }^{2}$ The composition symbol o will be suppressed throughout the paper.
} 
$u \in\left(\operatorname{lin}(F)^{\perp}\right)^{*}$. So we can view $u_{G}$ as an element in $\left(\operatorname{lin}(F)^{\perp}\right)^{*}$; it therefore defines a face $t(G, F)$ of $t(C, F)$. We can therefore define a map

$$
\begin{aligned}
t(\bullet, F):\{\text { faces } G \text { of } C \text { containing } F\} & \rightarrow\{\text { faces } H \text { of } t(C, F)\}, \\
G & \mapsto t(G, F)=t(C, F) \cap u_{G}^{\perp} .
\end{aligned}
$$

To check the bijectivity of $t(\bullet, F)$, first note that any face of $t(C, F)$ is defined by some element $u \in\left(\operatorname{lin}(F)^{\perp}\right)^{*}$ which can be viewed as an element in $V^{*}$ that vanishes on $\operatorname{lin}(F)$. Hence $u$ defines a face $G$ of $C$ containing $F$. Thus $t(\bullet, F)$ is surjective.

Next for two different faces $G_{1}, G_{2}$ containing $F$ defined by $u_{1}, u_{2} \in V^{*}$, there are vectors $v_{1}$ in $G_{1}$ and $v_{2}$ in $G_{2}$ such that $\left\langle u_{1}, v_{2}\right\rangle>0$ and $\left\langle u_{2}, v_{1}\right\rangle>0$. Thus $t\left(G_{1}, F\right)$ and $t\left(G_{2}, F\right)$ are different since the image of $v_{1}$ is not in $t\left(G_{2}, F\right)$ and the image of $v_{2}$ is not in $t\left(G_{1}, F\right)$. Hence the map $t(\bullet, F)$ is one-to-one. This gives the desired equation.

(C) follows from the definition of $t(C, F)$ since $\operatorname{lin}(C)=\operatorname{lin}(F) \oplus \operatorname{lin}(t(C, F))$.

We next verify the properties for lattice cones. For Item (di), by the definition of transverse lattice cones, the left hand side of the desired equation is

$$
t\left(\left(C, \Lambda_{C}\right),\left(F, \Lambda_{F}\right)\right)=\left(t(C, F), \Lambda_{t(C, F)}\right) .
$$

Similarly, the right hand side of the equation is

$$
\begin{aligned}
& t\left(t\left(\left(C, \Lambda_{C}\right),\left(F, \Lambda_{F}\right)\right), t\left(\left(F, \Lambda_{F}\right),\left(F^{\prime}, \Lambda_{F^{\prime}}\right)\right)\right) \\
= & t\left(\left(t\left(C, F^{\prime}\right), \Lambda_{t\left(C, F^{\prime}\right)}\right),\left(t\left(F, F^{\prime}\right), \Lambda_{t\left(F, F^{\prime}\right)}\right)\right) \\
= & \left(t\left(t\left(C, F^{\prime}\right), t\left(F, F^{\prime}\right)\right), \Lambda_{t\left(t\left(C, F^{\prime}\right), t\left(F, F^{\prime}\right)\right)}\right) .
\end{aligned}
$$

By Item (a), the first components of the two sides agree. On the other hand,

$$
\Lambda_{t(C, F)}=\pi_{F^{\perp}}\left(\Lambda_{C}\right)=\left(\pi_{t\left(F, F^{\prime}\right)^{\perp}} \pi_{F^{\prime}}\right)\left(\Lambda_{C}\right)=\pi_{t\left(F, F^{\prime}\right)}\left(\Lambda_{t\left(C, F^{\prime}\right)}\right)=\Lambda_{t\left(t\left(C, F^{\prime}\right), t\left(F, F^{\prime}\right)\right)} .
$$

Thus the second components of the two sides also agree. This proves Item (d).

For Item (e), the bijection in Eq. (7) can be enriched to the bijection

$$
\begin{aligned}
t(\bullet, F):\left\{\text { faces }\left(G, \Lambda_{G}\right) \text { of }\left(C, \Lambda_{C}\right) \text { containing }\left(F, \Lambda_{F}\right)\right\} & \rightarrow\left\{\text { faces }\left(H, \Lambda_{H}\right) \text { of }\left(t(C, F), \Lambda_{t(C, F)}\right\},\right. \\
\left(G, \Lambda_{G}\right) & \mapsto\left(t(G, F), \Lambda_{t(G, F)}\right)
\end{aligned}
$$

since we know from Proposition 2.21 that we can make sense of the lattice $\Lambda_{t(G, F)}$ of $t(G, F)$.

2.3. The coalgebra of lattice cones. Let us now introduce the concept of a connected coalgebra similar to that of a connected bialgebra [16]. See also [9, § 2.3].

Definition 2.23. Let $(\mathbf{C}, \Delta)$ be a coalgebra over a field $\mathbf{k}$ with counit $\varepsilon: \mathbf{C} \rightarrow \mathbf{k}$. It is called

(a) cograded if there is a grading $\mathbf{C}=\bigoplus_{n \geq 0} \mathbf{C}^{(n)}$ such that

$$
\Delta\left(\mathbf{C}^{(n)}\right) \subseteq \bigoplus_{p+q=n} \mathbf{C}^{(p)} \otimes \mathbf{C}^{(q)}, \quad n \geq 0 .
$$

Elements in $\mathbf{C}^{(n)}$ are said to have degree $n$.

(b) coaugmented if there is a linear map $u: \mathbf{k} \rightarrow \mathbf{C}$, called the coaugmentation, such that $\varepsilon u=\mathrm{id}_{\mathbf{k}}$.

(c) connected if $\mathbf{C}^{(0)}=\mathbf{k} u(1)$.

With the coaugmentation $u, \mathbf{C}$ is canonically isomorphic to ker $\varepsilon \oplus \mathbf{k} u(1)$. The proof of the following lemma is similar to the one for the case of connected bialgebras. 
Lemma 2.24. Let $(\mathbf{C}, \Delta)$ be a cograded, coaugmented, connnected coalgebra. Then

$$
\text { ker } \varepsilon=\bigoplus_{n \geq 1} \mathbf{C}^{(n)}
$$

\section{Further the reduced coproduct}

$$
\bar{\Delta}: \operatorname{ker} \varepsilon \rightarrow \operatorname{ker} \varepsilon \otimes \operatorname{ker} \varepsilon \quad x \mapsto \Delta(x)-x \otimes u(1)-u(1) \otimes x \quad \text { for all } x \in \mathbf{C},
$$

is well defined and $\bar{\Delta}^{m}\left(\mathbf{C}^{(n)}\right)=0$ for $m \geq n \geq 1$, where $\bar{\Delta}^{m}, m \geq 2$, is defined by the recursion $\bar{\Delta}^{m}=\left(\mathrm{id} \otimes \bar{\Delta}^{m-1}\right) \bar{\Delta}$.

The last condition is called the conilpotency of $\Delta[15]$.

We now equip the linear space $\mathbb{Q C}$ of lattice cones with the linear maps

$$
\begin{gathered}
\Delta: \mathbb{Q C} \longrightarrow \mathbb{Q C} \otimes \mathbb{Q C}, \quad\left(C, \Lambda_{C}\right) \longmapsto \sum_{F \leq C}\left(t(C, F), \Lambda_{t(C, F)}\right) \otimes\left(F, \Lambda_{F}\right), \\
\varepsilon: \mathbb{Q C} \longrightarrow \mathbb{Q}, \quad\left(C, \Lambda_{C}\right) \longmapsto \begin{cases}1, & C=\{0\}, \\
0, & C \neq\{0\},\end{cases}
\end{gathered}
$$

and

$$
u: \mathbb{Q} \longrightarrow \mathbb{Q} \mathfrak{C}, \quad 1 \longmapsto(\{0\},\{0\}) .
$$

Theorem 2.25. The quadruple (QC $, \Delta, \varepsilon, u)$ with $\Delta$, $\varepsilon$ and $u$ as in Eqs. (\$), (\$) and (10), defines a connected cograded coaugmented coalgebra with the grading

$$
\mathbb{Q} \mathfrak{C}=\bigoplus_{n \geq 0} \mathbb{Q} \mathfrak{C}^{(n)},
$$

where

$$
\mathfrak{C}^{(n)}:=\left\{\left(C, \Lambda_{C}\right) \in \mathfrak{C} \mid \operatorname{dim} C=n\right\}, \quad n \geq 0 .
$$

Proof. Let $\left(C, \Lambda_{C}\right)$ be a lattice cone in $\mathfrak{C}$, where $C \subset V_{k}$. On the one hand, we have

$$
\begin{aligned}
(\mathrm{id} \otimes \Delta) \Delta\left(C, \Lambda_{C}\right) & =\sum_{F \leq C}(\mathrm{id} \otimes \Delta)\left(\left(t(C, F), \Lambda_{t(C, F)}\right) \otimes\left(F, \Lambda_{F}\right)\right) \\
& =\sum_{F^{\prime} \leq F \leq C}\left(t(C, F), \Lambda_{t(C, F)}\right) \otimes\left(t\left(F, F^{\prime}\right), \Lambda_{t\left(F, F^{\prime}\right)}\right) \otimes\left(F^{\prime}, \Lambda_{F^{\prime}}\right) .
\end{aligned}
$$

On the other hand,

$$
\begin{aligned}
(\Delta \otimes \mathrm{id}) \Delta\left(C, \Lambda_{C}\right) & =\sum_{F^{\prime} \leq C}(\Delta \otimes \mathrm{id})\left(\left(t\left(C, F^{\prime}\right), \Lambda_{t\left(C, F^{\prime}\right)}\right) \otimes\left(F^{\prime}, \Lambda_{F^{\prime}}\right)\right) \\
& =\sum_{F^{\prime} \leq C} \sum_{H \leq t\left(C, F^{\prime}\right)}\left(\left(t\left(t\left(C, F^{\prime}\right), H\right), \Lambda_{t\left(t\left(C, F^{\prime}\right), H\right)}\right) \otimes\left(H, \Lambda_{H}\right)\right) \otimes\left(F^{\prime}, \Lambda_{F^{\prime}}\right) .
\end{aligned}
$$

For $H \leq t\left(C, F^{\prime}\right)$, by Proposition 2.22.(的) and (迆), there is $F \leq F^{\prime} \leq C$ such that $t\left(F, F^{\prime}\right)=H$ and $\Lambda_{t\left(F, F^{\prime}\right)}=\Lambda_{H}$. Further, since $\pi_{H^{\perp}} \pi_{F^{\prime}}=\pi_{t\left(F, F^{\prime}\right)^{\perp}} \pi_{F^{\perp}}=\pi_{F^{\perp}}$, we have

$$
t\left(t\left(C, F^{\prime}\right), H\right)=\pi_{H^{\perp}}\left(t\left(C, F^{\prime}\right)\right)=\pi_{H^{\perp}}\left(\pi_{F^{\prime}}(C)\right)=\pi_{F^{\perp}}(C)=t(C, F) .
$$

Similarly, $\Lambda_{t\left(t\left(C, F^{\prime}\right), H\right)}=\Lambda_{t(C, F)}$. This proves the coassociativity. It then follows from the definitions, that $\varepsilon$ is a counit for $\Delta$ and that $u$ yields a coaugmentation. Furthermore by Proposition 2.22.(可), the grading in Eq. (11) turns $(\mathbb{Q} \mathfrak{C}, \Delta, \varepsilon)$ into a cograded coalgebra. Since $\mathfrak{C}^{(0)}=$ $(\{0\},\{0\}), \mathbb{Q} \mathbb{C}$ is connected. 


\section{SUBDIVISION PROPERTIES}

In this section we study the behavior of linear maps on the space of lattice cones with respect to subdivisions.

\subsection{Subdivisions of lattice cones.}

Definition 3.1. (a) A subdivision of a cone $C$ is a set $\left\{C_{1}, \cdots, C_{r}\right\}$ of cones such that

(i) $C=\cup_{i=1}^{r} C_{i}$,

(ii) $C_{1}, \cdots, C_{r}$ have the same dimension as $C$, and

(iii) $C_{1}, \cdots, C_{r}$ intersect along their faces, i.e., $C_{i} \cap C_{j}$ is a face of both $C_{i}$ and $C_{j}$.

(b) A subdivision of a lattice cone $\left(C, \Lambda_{C}\right)$ is a set of lattice cones $\left\{\left(C_{i}, \Lambda_{C_{i}}\right) \mid 1 \leq i \leq r\right\}$ such that $\left\{C_{i} \mid 1 \leq i \leq r\right\}$ is a subdivision of $C$ and $\Lambda_{C_{i}}=\Lambda_{C}$ for all $1 \leq i \leq r$.

(c) A cone or lattice cone is called its own trivial subdivision.

Definition 3.2. Let $\left(C, \Lambda_{C}\right)$ be a simplicial lattice cone in $\mathfrak{C}_{k}$ and let $\underline{C}=\left\{\left(C_{1}, \Lambda_{C}\right), \cdots,\left(C_{r}, \Lambda_{C}\right)\right\}$ be a subdivision of $\left(C, \Lambda_{C}\right)$ into simplicial cones. Let $\mathcal{F}^{o}(\underline{C})$ denote the set of faces of $C_{1}, \cdots, C_{r}$ that are not contained in any proper face of $C$, that is, those faces of $C_{1}, \cdots, C_{r}$ that intersect with the interior of $C$.

Just as for ordinary cones, we have the following property.

Proposition 3.3. Any lattice cone can be subdivided into smooth lattice cones.

Proof. For a given lattice cone $\left(D, \Lambda_{C}\right)$ in a simplicial subdivision of a lattice cone $\left(C, \Lambda_{C}\right)$ with its primary generating set $\left\{v_{1}, \cdots, v_{n}\right\}$, we write $v_{i}=\sum_{j=1}^{n} a_{i j} u_{j}, a_{i j} \in \mathbb{Z}, i=1, \cdots, n$, where $\left\{u_{1}, \cdots, u_{n}\right\}$ is a basis of $\Lambda_{C}$. The absolute value of the determinant $w_{D}=\left|v_{1}, \cdots, v_{n}\right|:=\left|\operatorname{det}\left(a_{i j}\right)\right|$ lies in $\mathbb{Z}_{\geq 1}$ and is independent of the choice of a basis $\left\{u_{1}, \cdots, u_{n}\right\}$ of $\Lambda_{C}$. Further $w_{D}$ is equal to one if and only $\left(D, \Lambda_{C}\right)$ is smooth.

We now prove the proposition by contradiction. Suppose $\left(C, \Lambda_{C}\right)$ is a lattice cones that cannot be subdivided into smooth lattice cones. Then for any simplicial subdivision $\underline{C}:=\left\{\left(C_{i}, \Lambda_{C}\right)\right\}$ of $\left(C, \Lambda_{C}\right)$, we have

$$
w_{\underline{C}}:=\max \left\{w_{C_{i}}\right\}>1 \quad \text { and } \quad n_{\underline{C}}:=\max \left|\left\{i \mid, w_{C_{i}}=w_{\underline{C}}\right\}\right| \geq 1 .
$$

Choose a simplicial subdivision $\underline{C}$ of $\left(C, \Lambda_{C}\right)$ with $w_{\underline{C}}$ minimal and then among those, one with $n_{\underline{C}}$ minimal. We will construct a subdivision of $\left(C, \Lambda_{C}\right)$ that refines $\underline{C}$. Let $D=\left\langle v_{1}, \cdots, v_{n}\right\rangle$ be a cone in $\underline{C}$ with $w_{D}=w_{\underline{C}}$. Since $w_{D}>1$, the lattice cone $\left(D, \Lambda_{C}\right)$ is not smooth. So $\left\{v_{1}, \cdots v_{n}\right\}$ is not a lattice basis of $\Lambda_{C} \cap D$. Note that the set $\left\{v_{1}, \cdots, v_{n}\right\} \cup\left(\left(\sum_{i=1}^{n}[0,1) v_{i}\right) \cap \Lambda_{C}\right)$ spans $\Lambda_{C} \cap D$ as a monoid. So there is a vector $0 \neq v_{D}=\sum_{i=1}^{n} c_{i} v_{i} \in \Lambda_{C}$ with $c_{i} \in[0,1)$ rational.

Reordering $v_{i}$, we can assume that $c_{i} \neq 0$ for $i=1, \cdots, k$, and $c_{i}=0$ for $i=k+1, \cdots, n$. We now use the vector $v_{D}=\sum_{i=1}^{k} c_{i} v_{i}$ to subdivide the cones. Let $C_{i}=\left\langle v_{1}, \cdots, v_{k}, v_{k+1}^{i}, \cdots, v_{n}^{i}\right\rangle$, $i=1, \cdots, s$, be all the cones arising in the subdivision $\underline{C}$ that contain $\left\langle v_{1}, \cdots, v_{k}\right\rangle$ as a face, with $C_{1}=D$. Then the set of cones

$$
\left\{C_{i}, i>s\right\} \cup\left\{C_{i j}:=\left\langle v_{1}, \cdots, \check{v}_{j}^{D}, \cdots, v_{k}, v_{k+1}^{i}, \cdots, v_{n}^{i}\right\rangle \mid j=1, \ldots, k, i=1, \cdots, s\right\},
$$

where $\breve{v}_{j}^{D}$ means $v_{j}$ has been replaced by $v_{D}$, yields a new subdivision $\underline{C}^{\prime}$ of $C$. 
For elements in $\underline{C}^{\prime}$, the numbers $w_{C_{i}}, i>s$ coincide. For $i=1, \cdots, s$ and $j=1, \cdots, k$, $\left|v_{1}, \cdots, \check{v}_{j}^{D}, \cdots, v_{k}, v_{k+1}^{i}, \cdots, v_{n}^{i}\right|=c_{j}\left|v_{1}, \cdots, v_{k}, v_{k+1}^{i}, \cdots, v_{n}^{i}\right|<\left|v_{1}, \cdots, v_{k}, v_{k+1}^{i}, \cdots, v_{n}^{i}\right|=w_{C_{i}}$.

So $w_{C_{i j}}<w_{\underline{C}}$. Therefore either $w_{\underline{C}^{\prime}}<w_{\underline{C}}$, or $w_{\underline{C}^{\prime}}=w_{\underline{C}}$ and $n_{\underline{C}^{\prime}}<n_{\underline{C}}$. This gives the desired contradiction.

We collect the following facts before introducing more concepts on subdivisions of cones.

Lemma 3.4. Let $C$ be a cone and let $\underline{C}=\left\{C_{i}\right\}$ be a subdivision of $C$.

(a) If $F \leq C$ and $\operatorname{dim}(F)=\operatorname{dim}(C)$, then $F=C$.

(b) If $F \leq C$, then $F \cap C_{i} \leq C_{i}$.

(c) For any cone $G$ inside $C$, the $\operatorname{set} \underline{C}(G)=\left\{C_{i} \cap G \mid \operatorname{dim} C_{i} \cap G=\operatorname{dim} G\right\}$ is a subdivision of $G$.

Proof. (a) Let $F$ be defined by a linear functional $u$. Thus $\left.u\right|_{C} \geq 0$ and $F=C \cap u^{\perp}$. Since $u(F)=0$ we have $u(\operatorname{lin}(F))=0$. But $\operatorname{lin}(F)=\operatorname{lin}(C)$ since $\operatorname{dim}(F)=\operatorname{dim}(C)$. Thus $u(C)=0$, forcing $F=C$.

(b) If $F$ is defined by $u$, then $F \cap C_{i}=C \cap u^{\perp} \cap C_{i}=C_{i} \cap u^{\perp}$. So it is a face of $C_{i}$.

(C) Let $D$ be the union of $C_{i} \cap G$ with $\operatorname{dim} C_{i} \cap G<\operatorname{dim} G$. Then $G \backslash D$ is dense in $G$. Thus as its superset, the union of the cones $C_{i} \cap G$ with $\operatorname{dim} C_{i} \cap G=\operatorname{dim} G$ is dense in $G$ and hence is $G$. These cones intersect along their faces and hence provide a subdivision of $G$.

Given a subdivision $\underline{C}:=\left\{C_{1}, \cdots, C_{n}\right\}$ of $C$, set

$$
\mathcal{P}:=\mathcal{P}_{\underline{C}}:=\left\{\text { non-zero, proper face of some } C, C_{1}, \cdots, C_{n}\right\}
$$

and

$$
\mathcal{P}_{C}:=\{F \in \mathcal{P} \mid F \leq C\} .
$$

Denote $[n]:=\{1, \cdots, n\}$. For $I \subset[n]$, let

$$
C_{I}:=\cap_{i \in I} C_{i} \quad \text { and } \quad \mathcal{T}:=\left\{C_{I} \mid \emptyset \neq I \subset[n]\right\} .
$$

For a face $F \in \mathcal{P}$, set

$$
J(F):=\left\{i \in[n] \mid F \leq C_{i}\right\} \quad \text { and } \quad j(F):=|J(F)| .
$$

Note that $H \preceq F$ implies $J(H) \supset J(F)$.

For any subset $Q$ of $\mathcal{P}$ and $i \geq 0$, we further set

$$
Q_{i}:=\{F \in Q \mid j(F)=i\}, \quad Q_{\geq i}:=\{F \in Q \mid j(F) \geq i\} .
$$

In particular this notation applies to $\mathcal{P}_{C}$.

Definition 3.5. Let $\left\{C_{i}\right\}$ be a subdivision of $C$. A proper face of a $C_{i}$ is called a subdivision induced face (SIF) if it arises as a cone in a nontrivial subdivision of some face of $C$.

Distinguishing between faces induced and not induced by a subdivision,

$$
\mathcal{P}_{\mathrm{SI}}:=\{F \in \mathcal{P} \mid F \text { is an SIF }\} \quad \text { and } \quad \mathcal{P}_{N}:=\{F \in \mathcal{P} \mid F \npreceq C, F \text { is not an SIF }\} .
$$

yield a partition

$$
\mathcal{P}=\mathcal{P}_{C, 0} \coprod \mathcal{P}_{C, 1} \coprod \mathcal{P}_{C, \geq 2} \coprod \mathcal{P}_{\mathrm{SI}} \coprod \mathcal{P}_{N}
$$

of $\mathcal{P}$ into the five subsets of cones arising respectively as proper nonzero faces

- of $C$ that are not faces of any cone in the subdivision, 
- of $C$ that are faces of exactly one cone in the subdivision,

- of $C$ that are faces of at least two cones in the subdivision,

- of some $C_{i}$ and arising from a nontrivial subdivision of some face of $C$,

- of some $C_{i}$ but not of $C$ and not arising from a nontrivial subdivision of any face of $C$.

Example 3.6. For the subdivision $\left\{\left\langle e_{1}, e_{1}+e_{2}, e_{3}\right\rangle,\left\langle e_{2}, e_{1}+e_{2}, e_{3}\right\rangle\right\}$ of the cone $\left\langle e_{1}, e_{2}, e_{3}\right\rangle$, we have

$$
\begin{gathered}
\mathcal{P}_{C, 0}=\left\{\left\langle e_{1}, e_{2}\right\rangle\right\} ; \mathcal{P}_{C, 1}=\left\{\left\langle e_{1}\right\rangle,\left\langle e_{1}, e_{3}\right\rangle,\left\langle e_{2}\right\rangle,\left\langle e_{2}, e_{3}\right\rangle\right\} ; \mathcal{P}_{C, \geq 2}=\left\{\left\langle e_{3}\right\rangle\right\} ; \\
\mathcal{P}_{\mathrm{SI}}=\left\{\left\langle e_{1}, e_{1}+e_{2}\right\rangle,\left\langle e_{2}, e_{1}+e_{2}\right\rangle\right\} ; \mathcal{P}_{N}=\left\{\left\langle e_{1}+e_{2}\right\rangle,\left\langle e_{1}+e_{2}, e_{3}\right\rangle\right\} .
\end{gathered}
$$

Lemma 3.7. (a) The relation

$$
R:=\left\{(F, G) \in \mathcal{P}_{\mathrm{SI}} \times \mathcal{P}_{C, 0} \mid F \subset G, \operatorname{dim} F=\operatorname{dim} G\right\}
$$

defines a surjective map

$$
\begin{aligned}
\alpha: \mathcal{P}_{\mathrm{SI}} & \rightarrow \mathcal{P}_{C, 0} \\
F & \longmapsto G,(F, G) \in R .
\end{aligned}
$$

(b) For each $G \in \mathcal{P}_{C, 0}$, the set $\alpha^{-1}(G)$ is a subdivision of $G$.

Proof. (a) Let $F \in \mathcal{P}_{\mathrm{SI}}$. Then $F$ arises in a subdivision of a face $G$ of $C$, but is not equal to $G$. Such a face $G$ of $C$ is unique: if $F$ is contained in $G_{1}$ and $G_{2}$, then

$$
\operatorname{dim} G_{i} \geq \operatorname{dim}\left(G_{1} \cap G_{2}\right) \geq \operatorname{dim} F \geq \operatorname{dim} G_{i}, i=1,2 .
$$

Thus $\operatorname{dim}\left(G_{1} \cap G_{2}\right)=\operatorname{dim} G_{1}=\operatorname{dim} G_{2}$. Also $G_{1} \cap G_{2} \leq G_{1}, G_{1} \cap G_{2} \leq G_{2}$. So $G_{1}=G_{1} \cap G_{2}=G_{2}$ by Lemma 3.4 (D). Further $G$ lies in $\mathcal{P}_{C, 0}$ for, if $G$ were contained in some $C_{i}$, then $G=G \cap C_{i}$ would be a face of $C_{i}$ by Lemma 3.4.(何), leading to a contradiction. Thus we obtain a map

$$
\alpha: \mathcal{P}_{\mathrm{SI}} \rightarrow \mathcal{P}_{C, 0}
$$

sending $F \in \mathcal{P}_{\text {SI }}$ to the unique face $G$ of $C$ above. The map is surjective in view of Lemma B.4.(C).

(b) For $G \in \mathcal{P}_{C, 0}, \alpha^{-1}(G)$ gives the subdivision of $F$ induced by $\left\{C_{i}\right\}$ as explicited in Lemma 3.4.(C).

On the grounds of this lemma we introduce further useful notations. For $G \in \mathcal{P}_{C, 0}$, let

$$
\alpha^{-1}(G)=\left\{F_{1}^{G}, \cdots, F_{\ell(G)}^{G}\right\}, \quad \ell(G)=\left|\alpha^{-1}(G)\right| .
$$

For $k \geq 1$, let $\alpha^{-1}(G)_{k}=\left\{F^{G} \in \alpha^{-1}(G) \mid j\left(F^{G}\right)=k\right\}$.

3.2. Induced subdivisions on transverse cones. We now study how a subdivision of a cone induces a subdivision on a transverse cone. We first recall the following fact.

Lemma 3.8. (Separation Lemma [可]) For cones $C_{1}$ and $C_{2}$ with $C_{1} \cap C_{2} \leq C_{1}$ and $C_{1} \cap C_{2} \leq C_{2}$, there exists a linear function $u$ such that $\left.u\right|_{C_{1}} \geq 0,\left.u\right|_{C_{2}} \leq 0$ and $C_{1} \cap C_{2}=C_{1} \cap u^{\perp}=C_{2} \cap u^{\perp}$.

Applying the separation lemma to transverse cones yields

Lemma 3.9. (a) Let $C_{1}$ and $C_{2}$ be cones and let $F:=C_{1} \cap C_{2}$. If $F \supsetneqq C_{1}$ and $F \supsetneqq C_{2}$, then $t\left(C_{1}, F\right) \neq t\left(C_{2}, F\right)$.

(b) Let $\left\{C_{i}\right\}$ be a subdivision of $C$ and let $H \in \mathcal{P}$. Then the cones $\left\{t\left(C_{i}, H\right) \mid i \in J(H)\right\}$ are distinct. 
Proof. (a) Take the linear function $u$ in Lemma 3.8. By assumption, there are $c_{1} \in C_{1}$ and $c_{2} \in C_{2}$ such that $u\left(c_{1}\right)>0$ and $u\left(c_{2}\right)<0$. Thus $C_{1}$ and $C_{2}$ are distinct. Since $u$ vanishes on $F, u$ descends to a linear function $\bar{u}$ on the space $\operatorname{lin}(F)^{\perp}$. Further, we have $\bar{u}\left(c_{1}+\operatorname{lin} F\right)=u\left(c_{1}\right)$ and $\bar{u}\left(c_{2}+\operatorname{lin} F\right)=u\left(c_{2}\right)$. Since $c_{1}+\operatorname{lin} F \in t\left(C_{1}, F\right)$ and $c_{2}+\operatorname{lin} F \in t\left(C_{2}, F\right)$, the conclusion follows.

(b) Given $H \in \mathcal{P}$, let $m \neq n$ be in $J(H)$. Since $\left\{C_{i}\right\}$ is a subdivision of $C$, the condition in Item (a) is satisfied. Thus $t\left(C_{m}, C_{m} \cap C_{n}\right)$ and $t\left(C_{n}, C_{m} \cap C_{n}\right)$ are distinct. But $t\left(C_{m}, C_{m} \cap C_{n}\right)$ and $t\left(C_{n}, C_{m} \cap C_{n}\right)$ are quotients of $t\left(C_{m}, H\right)$ and $t\left(C_{n}, H\right)$ respectively modulo $\operatorname{lin}\left(C_{m} \cap C_{n}\right)$. Hence $t\left(C_{m}, C_{m} \cap C_{n}\right)$ and $t\left(C_{n}, C_{m} \cap C_{n}\right)$ are also distinct.

Lemma 3.10. Let $\left\{C_{1}, \cdots, C_{n}\right\}$ be a subdivision of $C$ and let $F$ be a face of some $C_{i}$.

(a) The cones $\left\{t\left(C_{i}, F\right) \mid i \in J(F)\right\}$ are distinct and form a subdivision of $t(C, F)$. Here by $t(C, F)$ we mean the projection of $C$ in $\operatorname{lin}(F)^{\perp}$ even if $F$ is not a face of $C$. In particular, if $F$ is in $\mathcal{P}_{C, 1}$, so that $F \leq C$ and $J(F)=\left\{C_{i_{0}}\right\}$, then $t(C, F)=t\left(C_{i_{0}}, F\right)$.

(b) For $I \subset J(F)$ we have

$$
\bigcap_{i \in I} t\left(C_{i}, F\right)=t\left(C_{I}, F\right)
$$

(c) If $F \in \mathcal{P}_{N}$, that is, if $F$ is a face of a $C_{i}$ but neither a face of $C$ nor an SIF, then $t(C, F)$ contains a line.

Proof. (国) Clearly, $t(C, F)=\bigcup_{i=1}^{n} t\left(C_{i}, F\right)$. We first need to prove that $t(C, F)=\bigcup_{i \in J(F)} t\left(C_{i}, F\right)$.

For any $x$ in $C_{i}$ such that $i \notin J(F)$, let $x_{0} \neq x$ be any point in the relative interior of $F \cap C_{i}$. The line segment $\left[x_{0}, x\right]$ lies in $C_{i}$ and hence in $C$. It intersects $C_{j}$ for some $j \in J(F)$ at a point $y \neq x_{0}$ for otherwise, $x_{0}=y \in C_{j}$ would lie on a face of $C_{j}, j \notin J(F)$, so the relative interior of $F \cap C_{i}$ would lie on a face of $C_{j}$ contradicting the assumption on $J(F)$. As an element of $V_{k}$, we have

$$
x=\frac{\left\|x-x_{0}\right\|}{\left\|y-x_{0}\right\|}\left(y-x_{0}\right)+x_{0}
$$

Therefore

$$
\pi_{F^{\perp}}(x)=\pi_{F^{\perp}}\left(\frac{\left\|x-x_{0}\right\|}{\left\|y-x_{0}\right\|} y\right),
$$

which is an element of $\bigcup_{i \in J(F)} t\left(C_{i}, F\right)$, as required.

We next prove that the cones $t\left(C_{i}, F\right), i \in J(F)$, only intersect along their faces. If distinct cones $C_{i}$ and $C_{j}, i, j \in[n]$ have a common face $F$, then $F \subset C_{i} \cap C_{j}$. By Lemma 3.8, there exists a linear function $u$, such that $\left.u\right|_{C_{i}} \geq 0,\left.u\right|_{C_{j}} \leq 0$ and $C_{i} \cap C_{j}=C_{i} \cap u^{\perp}=C_{j} \cap u^{\perp}$. Then for $x_{i} \in C_{i}, x_{j} \in C_{j}$, if $\pi_{k, F^{\perp}}\left(x_{i}\right)=\pi_{k, F^{\perp}}\left(x_{j}\right)$, then $u\left(x_{i}\right)=u\left(x_{j}\right)$, so $u\left(x_{i}\right)=u\left(x_{j}\right)=0$. Therefore $x_{i} \in C_{i} \cap C_{j}$ and $x_{j} \in C_{i} \cap C_{j}$. So $t\left(C_{i}, F\right) \cap t\left(C_{j}, F\right)=t\left(C_{i} \cap C_{j}, F\right)$. This gives what we need since by Proposition 2.22.(D), the right hand side is a face of the two cones on the left hand side. Now assertion (a) follows from Lemma 3.9 (b).

(D) We proceed by induction on $|I|$. The case $|I|=1$ is trivial. Reordering the cones if necessary, we assume that the desired equation holds for $I=[k]$ with $k \geq 1$, and aim to prove it when $I=[k+1]$. If $C_{[k]} \subset C_{k+1}$, then $C_{[k]}=C_{[k+1]}$ and $t\left(C_{[k]}, F\right) \subseteq t\left(C_{[k+1]}, F\right)$. Thus

$$
t\left(C_{[k+1]}, F\right)=t\left(C_{[k]}, F\right)=t\left(C_{[k]}, F\right) \cap t\left(C_{k+1}, F\right)=\cap_{i \in[k+1]} t\left(C_{i}, F\right) .
$$

If $C_{[k]} \not \subset C_{k+1}$, then we can apply the same argument as in the previous item with $C_{m}, C_{n}$ replaced by $C_{[k]}, C_{k+1}$ since the argument only requires the two cones to be different and to intersect along 
their faces. It follows that $t\left(C_{[k]}, F\right) \cap t\left(C_{k+1}, F\right)=t\left(C_{[k]} \cap C_{k+1}, F\right)$, as needed to complete the induction.

(C) We prove the property by induction on $k=\operatorname{dim}(C)$.

For $k=1$, there is nothing to prove. Let us assume that the claim holds for $k=n$ and let us prove it for $k=n+1$.

Let $F$ be a proper face of a $C_{i}$ but is neither a face of $C$ nor an SIF. Since $F$ is not a face of $C$, it is either not contained in any proper face of $C$, or it is properly contained in a proper face of $C$.

Assume $F$ is not contained in any proper face of $C$. Then there exists a point $x_{0}$ of $F$ that is in the relative interior of $C$. Since $\operatorname{dim}(F)<\operatorname{dim}(C)$, there is a point $x_{1} \in C, x_{1} \neq x_{0}$, such that $\pm x_{1}+x_{0} \in \operatorname{lin}^{\perp}(F)$. Therefore $t(C, F)$ contains the line $\mathbb{R} x_{1}$.

Now assume that $F$ is properly contained in a proper face of $C$. Let $G$ be a face of $C$ that contains $F$ and has minimal dimension with this property. This $G$ is unique; indeed if both $G_{1}$ and $G_{2}$ are faces of $C$ containing $F$ and having minimal dimension, then so is $G_{1} \cap G_{2}$, which by Lemma 3.4.(D) implies that $G_{1}=G_{2}=G_{1} \cap G_{2}$, leading to a contradiction. Now $F$ is neither a face of $G$ nor an SIF, so by the induction hypothesis, $t(G, F)$ contains a line. Then $t(C, F) \supseteq t(G, F)$ contains a line.

3.3. Compatibility of the convolution product with subdivisions. Let $C$ a class of sets stable under finite intersections and finite unions. A map $\varphi$ on $C$ with values in a commutative algebra $A$ is said to satisfy the valuation property if

$$
\varphi(A \cup B)+\varphi(A \cap B)=\varphi(A)+\varphi(B) \text { for all } A, B \in C .
$$

A straightforward induction shows that a map obeys the valuation property if and only if it satisfies the following compatibility with unions:

$$
\phi\left(\cup_{i=1}^{n} A_{i}\right)=\sum_{\emptyset \neq I \subset[n]}(-1)^{|I|-1} \phi\left(A_{I}\right) \quad \text { for all } A_{1}, \cdots, A_{n} \in C,
$$

where we have set $A_{I}:=\cap_{i \in I} A_{i}$. For the cardinal on finite sets, Eq. (18) amounts to the inclusionexclusion principle.

We extend the valuation property of the form in Eq. (18) to subdivision properties for maps on lattice cones. Notice that that the set of lattice cones is only equipped with a partial intersection and a partial union.

Definition 3.11. A linear map $\phi$ on $\mathbb{Q} \mathfrak{C}$ with values in a commutative algebra has

- the discrete closed subdivision property if for a lattice cone $\left(C, \Lambda_{C}\right)$ and its subdivision $\underline{C}=\left\{\left(C_{i}, \Lambda_{C_{i}}\right)\right\}_{i=1, \cdots, n}$,

$$
\phi\left(C, \Lambda_{C}\right)=\sum_{\emptyset \neq I \subseteq[n]}(-1)^{|I|-1} \phi\left(C_{I}, \Lambda_{C_{I}}\right) .
$$

- the discrete open subdivision property if for a lattice cone $\left(C, \Lambda_{C}\right)$ and its subdivision $\underline{C}=\left\{\left(C_{i}, \Lambda_{C_{i}}\right)\right\}_{i=1, \cdots, n}$,

$$
\phi\left(C, \Lambda_{C}\right)=\sum_{F \in \mathcal{F}^{o}(\underline{C})} \phi\left(F, \Lambda_{F}\right) .
$$


- the continuous subdivision property if for a lattice cone $\left(C, \Lambda_{C}\right)$ and its subdivision $\underline{C}=\left\{\left(C_{i}, \Lambda_{C_{i}}\right)\right\}_{i=1, \cdots, n}$,

$$
\phi\left(C, \Lambda_{C}\right)=\sum_{i=1}^{n} \phi\left(C_{i}, \Lambda_{C_{i}}\right) .
$$

The discrete closed subdivision property is closely related to discrete open subdivision property. For a linear map $\phi: \mathbb{Q} \mathfrak{C} \rightarrow \mathcal{A}$, we define the map $\phi^{c}: \mathbb{Q} \mathfrak{C} \rightarrow \mathcal{A}$ by

$$
\phi^{c}\left(C, \Lambda_{C}\right):=\sum_{F \leqslant C} \phi\left(F, \Lambda_{F}\right)
$$

Then we have

Proposition 3.12. A linear map $\phi$ has the discrete open subdivision property if and only if $\phi^{c}$ has the discrete closed subdivision property.

Proof. For a lattice cone $\left(C, \Lambda_{C}\right)$ and its subdivision $\underline{C}=\left\{\left(C_{i}, \Lambda_{C_{i}}\right)\right\}_{i=1, \cdots, n}$,

$$
\begin{aligned}
\phi^{c}(C)-\sum_{\emptyset \neq I \subseteq[n]}(-1)^{|I|-1} \phi^{c}\left(C_{I}\right) & =\sum_{F \preccurlyeq C} \phi(C)-\sum_{\emptyset \neq I \subseteq[n]}(-1)^{|I|-1} \sum_{F \preccurlyeq C_{I}} \phi(F) \\
& =\sum_{F \preccurlyeq C} \phi(F)-\sum_{F \in \mathcal{F}^{c}(\underline{C})}\left(\sum_{\emptyset \neq I \subseteq J(F)}(-1)^{|I|-1}\right) \phi(F) \\
& =\sum_{F \preccurlyeq C} \phi(F)-\sum_{F \in \mathcal{F}^{c}(\underline{C})} \phi(F) \\
& =\phi(C)-\sum_{F \in \mathcal{F}^{o}(\underline{C})} \phi(F)+\sum_{G \precsim C}\left(\phi(G)-\sum_{F \in \underline{C}_{G}} \phi(F)\right) \\
& =\phi(C)-\sum_{F \in \mathcal{F}^{o}(\underline{C})} \phi(F)+\sum_{G \precsim C}\left(\phi(G)-\sum_{F \in \mathcal{F}^{o}(\underline{C}(G))} \phi(F)\right) .
\end{aligned}
$$

Here the third equation follows from $\sum_{Y \subseteq X}(-1)^{|Y|}=0$ for a finite set $X$; the fourth equation follows from

$$
\underline{C}_{G}:=\left\{F \in \mathcal{F}^{c}(\underline{C}) \mid F \leqslant G, F \text { is not contained in any proper face of } G\right\},
$$

and the fact that $\mathcal{F}^{c}(\underline{C})$ is a disjoint union of $\underline{C}_{G}, G \leqslant C$; the fifth equation is a consequence of Lemma 3.4 (c).

Now if $\phi$ has the discrete open subdivision property, then the right hand side is zero, so the left hand is zero and $\phi^{c}$ has the discrete closed subdivision property.

Conversely, if $\phi^{c}$ has the discrete closed subdivision property. Then the left hand side, and hence the right hand side, is zero for all $C$. Note that for a one dimensional cone, the second sum on the right hand side is zero, showing that $\phi$ has the discrete open subdivision property for one dimensional cone. Then by an induction on the dimension, $\phi$ is discrete open subdivision property for all cones.

We now state our main theorem on discrete closed subdivision property of convolution quotient of linear maps on lattice cones.

Theorem 3.13. Let $\phi$ and $\psi$ be linear maps on $\mathbb{Q} \mathfrak{C}$ with values in a commutative algebra $A$ that satisfy the following properties: 
(a) $\phi$ and $\psi$ satisfy the discrete closed subdivision property property;

(b) $\phi(\{0\},\{0\})=\psi(\{0\},\{0\})=1$;

(c) for a lattice cone $\left(C, \Lambda_{C}\right)$ that is not strongly convex, $\phi\left(\left(C, \Lambda_{C}\right)\right)=\psi\left(\left(C, \Lambda_{C}\right)\right)=0$.

Then the convolution quotient $\chi:=\phi^{*(-1)} * \psi$ has the continuous subdivision property.

We introduce more notations and preliminary results before actually proving the theorem. In the coalgebra $\mathbb{Q} \mathfrak{C}$, we set

$$
\Delta^{\prime}\left(C, \Lambda_{C}\right):=\Delta\left(C, \Lambda_{C}\right)-(\{0\},\{0\}) \otimes\left(C, \Lambda_{C}\right)-\left(C, \Lambda_{C}\right) \otimes(\{0\},\{0\}) .
$$

We also use $*^{\prime}$ to denote the restricted product of the convolution product in the space $\mathcal{L}(\mathbb{Q} C, A)$ of linear maps built from $\Delta^{\prime}$, that is

$$
\phi_{1} *^{\prime} \phi_{2}=m_{A}\left(\phi_{1} \otimes \phi_{2}\right) \Delta^{\prime}
$$

where $m_{A}$ is the multiplication of $A$. Then $\phi_{1} * \phi_{2}=\phi_{1} *^{\prime} \phi_{2}+\phi_{1}+\phi_{2}$.

Lemma 3.14. The map $\chi$ satisfies the recursive formula

$$
\chi=\psi-\phi-\phi *^{\prime} \chi
$$

Proof. The right hand side of the equation gives

$$
\psi-\phi-\phi *^{\prime}\left(\phi^{*(-1)} * \psi\right)=\psi-\phi-\phi *\left(\phi^{*(-1)} * \psi\right)+\phi+\phi^{*(-1)} * \psi=\chi,
$$

as needed.

Now, with $\mathcal{P}$ as defined in Eq. (12), we have

$$
\Delta^{\prime}\left(\left(C, \Lambda_{C}\right)-\sum_{i=0}^{n}\left(C_{i}, \Lambda_{C_{i}}\right)\right)=\sum_{F \in \mathcal{P}} c(F) \otimes\left(F, \Lambda_{F}\right)
$$

where $c(F):=\sum_{i=0}^{n} c_{i}(F)$ while, with the convention that $C_{0}=C$,

$$
c_{i}(F):= \begin{cases}\left(t\left(C_{i}, F\right), \Lambda_{t\left(C_{i}, F\right)}\right), & i=0, F \leq C_{0}, \\ -\left(t\left(C_{i}, F\right), \Lambda_{t\left(C_{i}, F\right)}\right), & i=1, \cdots, n, F \leq C_{i}, \\ 0, & F \npreceq C_{i} .\end{cases}
$$

Then by Eq. (23), we have

$$
\chi\left(\left(C, \Lambda_{C}\right)-\sum_{i=1}^{n}\left(C_{i}, \Lambda_{C_{i}}\right)\right)=(\psi-\phi)\left(\left(C, \Lambda_{C}\right)-\sum_{i=1}^{n}\left(C_{i}, \Lambda_{C_{i}}\right)\right)-\sum_{F \in \mathcal{P}} \phi(c(F)) \chi\left(F, \Lambda_{F}\right) .
$$

Let $\mathcal{P}([n])$ denote the power set of $[n]$. Consider the surjective map

$$
\lambda: \mathcal{P}([n]) \backslash \emptyset \longrightarrow \mathcal{T}, \quad I \mapsto C_{I} .
$$

For $H \in \mathcal{T}$, denote

$$
\lambda_{H}:=\sum_{J \in \lambda^{-1}(H)}(-1)^{|J|-1} .
$$

Then the discrete closed subdivision property of $\phi$ in Eq. (19) can be expressed as

$$
\phi\left(C, \Lambda_{C}\right)=\sum_{H \in \mathcal{T}} \lambda_{H} \phi\left(H, \Lambda_{H}\right) .
$$


Likewise, for $H \in \mathcal{P}$ and the subdivision $\left\{t\left(C_{i}, H\right) \mid i \in J(H)\right\}$ of $t(C, H)$ in Lemma 3.10.(国), the discrete closed subdivision property for this subdivision is

$$
\begin{aligned}
\phi\left(t(C, H), \Lambda_{t(C, H)}\right) & =\sum_{I \subset J(H)}(-1)^{|I|-1} \phi\left(\bigcap_{i \in I} t\left(C_{i}, H\right), \Lambda_{t(C, H)} \cap \operatorname{lin}\left(\bigcap_{i \in I} t\left(C_{i}, H\right)\right)\right) \\
& =\sum_{I \subset J(H)}(-1)^{|I|-1} \phi\left(t\left(C_{I}, H\right), \Lambda_{t(C, H)} \cap \operatorname{lin}\left(t\left(C_{I}, H\right)\right)\right)
\end{aligned}
$$

by Lemma 3.10.(B). Furthermore, for the lattices on the right hand side, we have

$$
\Lambda_{t(C, H)} \cap \operatorname{lin}\left(t\left(C_{I}, H\right)\right)=\pi_{H^{\perp}}\left(\Lambda_{C}\right) \cap \operatorname{lin}\left(\pi_{H^{\perp}}\left(C_{I}\right)\right)=\pi_{H^{\perp}}\left(\Lambda_{C}\right) \cap \pi_{H^{\perp}}\left(\operatorname{lin}\left(C_{I}\right)\right)
$$

which agrees with

$$
\Lambda_{t\left(C_{I}, H\right)}=\pi_{H^{\perp}}\left(\Lambda_{C_{I}}\right)=\pi_{H^{\perp}}\left(\Lambda_{C} \cap \operatorname{lin}\left(C_{I}\right)\right)
$$

by Lemma 2.20. Therefore, the discrete closed subdivision property in Eq. (26) becomes

$$
\phi\left(t(C, H), \Lambda_{t(C, H)}\right)=\sum_{I \subset J(H)}(-1)^{|I|-1} \phi\left(t\left(C_{I}, H\right), \Lambda_{t\left(C_{I}, H\right)}\right)=\sum_{F \in \mathcal{T}(H)} \lambda_{F} \phi\left(t(F, H), \Lambda_{t(F, H)}\right),
$$

where we have set

$$
\mathcal{T}(H):=\left\{C_{I} \mid I \subseteq J(H)\right\} .
$$

Then with the notation in Eq. (14), we set

$$
\mathcal{T}_{\ell}(H):=\left\{C_{I} \mid I \subseteq J(H), j\left(C_{I}\right)=\ell\right\} .
$$

Now we are ready to state the key combinatorial facts for the proof of Theorem 3.13 .

Proposition 3.15. With the above notations, the following equations hold for $\ell \geq 2$.

$$
\begin{aligned}
& \sum_{F \in \mathcal{P}_{\ell}}\left(\phi(c(F))-\sum_{m=2}^{\ell-1} \sum_{H \in \mathcal{T}_{m}(F)} \lambda_{H} \phi\left(t(H, F), \Lambda_{t(H, F)}\right)\right) \chi\left(F, \Lambda_{F}\right) \\
= & (\psi-\phi)\left(\sum_{F \in \mathcal{T}_{\ell}} \lambda_{F}\left(F, \Lambda_{F}\right)\right)-\sum_{G \in \mathcal{P}_{C, 0}} \sum_{H \in \alpha^{-1}(G)} \phi\left(t(F, H), \Lambda_{t(F, H)}\right) \chi\left(H, \Lambda_{H}\right) \\
& -\sum_{k \geq \ell+1} \sum_{H \in \mathcal{P}_{k}} \sum_{F \in \mathcal{T}_{\ell}(H)} \lambda_{F} \phi\left(t(F, H), \Lambda_{t(F, H)}\right) \chi\left(H, \Lambda_{H}\right) .
\end{aligned}
$$

$\chi\left(\left(C, \Lambda_{C}\right)-\sum_{i}\left(C_{i}, \Lambda_{C_{i}}\right)\right)=(\psi-\phi)\left(\left(C, \Lambda_{C}\right)-\sum_{i}\left(C_{i}, \Lambda_{C_{i}}\right)-\sum_{m=2}^{\ell-1} \sum_{F \in \mathcal{T}_{m}} \lambda_{F}\left(F, \Lambda_{F}\right)\right)$

$$
\begin{aligned}
& -\sum_{G \in \mathcal{P}_{C, 0}} \phi\left(t(C, G), \Lambda_{t(C, G)}\right)\left(\chi\left(G, \Lambda_{G}\right)-\sum_{m=1}^{\ell-1} \sum_{H \in \alpha^{-1}(G)_{m}} \chi\left(H, \Lambda_{H}\right)\right) \\
& -\sum_{k \geq \ell} \sum_{F \in \mathcal{P}_{k}}\left(\phi(c(F))-\sum_{m=2}^{\ell-1} \sum_{H \in \mathcal{T}_{m}(F)} \lambda_{H} \phi\left(t(H, F), \Lambda_{t(H, F)}\right)\right) \chi\left(F, \Lambda_{F}\right) .
\end{aligned}
$$


Proof. Proof of Eq. (30): We have $\mathcal{P}_{\ell}=\mathcal{T}_{\ell} \amalg \mathcal{P}_{\ell}^{\prime}$ where $\mathcal{P}_{\ell}^{\prime}:=\mathcal{P}_{\ell} \backslash \mathcal{T}_{\ell}$.

We first consider the partial sum

$$
\sum_{F \in \mathcal{T}_{\ell}}\left(\phi(c(F))-\sum_{m=2}^{\ell-1} \sum_{H \in \mathcal{T}_{m}(F)} \lambda_{H} \phi\left(t(H, F), \Lambda_{t(H, F)}\right)\right) \chi\left(F, \Lambda_{F}\right)
$$

on the left hand side of Eq. (30) over the subset $\mathcal{T}_{\ell}$ of $\mathcal{P}_{\ell}$ introduced in (29). For $F \in \mathcal{T}_{\ell}$, we have $F=\cap_{i \in J(F)} C_{i}$ with $j(F)=\ell$ and thus $\mathcal{T}_{\ell}(F)=\{F\}$. Since $\ell \geq 2$, we have the disjoint union

$$
\mathcal{T}_{\ell}=\left(\mathcal{T}_{\ell} \cap \mathcal{P}_{C, \geq 2}\right) \amalg\left(\mathcal{T}_{\ell} \cap \mathcal{P}_{\mathrm{SI}}\right) \amalg\left(\mathcal{T}_{\ell} \cap \mathcal{P}_{N}\right)
$$

and

$$
\mathcal{P}_{\ell}^{\prime}=\left(\mathcal{P}_{\ell}^{\prime} \cap \mathcal{P}_{C, \geq 2}\right) \amalg\left(\mathcal{P}_{\ell}^{\prime} \cap \mathcal{P}_{\mathrm{SI}}\right) \amalg\left(\mathcal{P}_{\ell}^{\prime} \cap \mathcal{P}_{N}\right)=\mathcal{P}_{C, \geq 2}^{\prime} \amalg \mathcal{P}_{\mathrm{SI}}^{\prime} \amalg \mathcal{P}_{N}^{\prime}
$$

Then by Eq. (27) we have

$$
\begin{gathered}
\phi(c(F))-\sum_{m=2}^{\ell-1} \sum_{H \in \mathcal{T}_{m}(F)} \lambda_{H} \phi\left(t(H, F), \Lambda_{t(H, F)}\right) \\
= \begin{cases}\lambda_{F} \phi(t(F, F),\{0\})=\lambda_{F}, & \text { for } F \in \mathcal{T}_{\ell} \cap \mathcal{P}_{C, \geq 2}, \\
-\phi\left(t(C, F), \Lambda_{t(C, F)}\right)+\lambda_{F} \phi(t(F, F),\{0\}) & \text { for } F \in \mathcal{T}_{\ell} \cap \mathcal{P}_{\mathrm{SI}}, \\
=-\phi\left(t(C, F), \Lambda_{t(C, F)}\right)+\lambda_{F}, & \text { for } F \in \mathcal{T}_{\ell} \cap \mathcal{P}_{N} .\end{cases}
\end{gathered}
$$

where in the last case we have used $\phi\left(t(C, F), \Lambda_{t(C, F)}\right)=0$ as a consequence of Lemma 3.10.(何). Therefore we have

$$
\begin{aligned}
& \sum_{F \in \mathcal{T}_{\ell}}\left(\phi(c(F))-\sum_{m=2}^{\ell-1} \sum_{H \in \mathcal{T}_{m}(F)} \lambda_{H} \phi\left(t(H, F), \Lambda_{t(H, F)}\right)\right) \chi\left(F, \Lambda_{F}\right) \\
= & -\sum_{G \in \mathcal{P}_{C, 0}} \sum_{F \in \alpha^{-1}(G) \cap \mathcal{T}} \phi\left(t(C, G), \Lambda_{t(C, G)}\right) \chi\left(F, \Lambda_{F}\right)+\sum_{F \in \mathcal{T}_{\ell}} \lambda_{F} \chi\left(F, \Lambda_{F}\right) .
\end{aligned}
$$

By definition, the second term on the right hand side of the above equation reads

$$
(\psi-\phi)\left(\sum_{F \in \mathcal{T}_{\ell}} \lambda_{F}\left(F, \Lambda_{F}\right)\right)-\sum_{(F, H) \in U_{\ell}} \lambda_{F} \phi\left(t(F, H), \Lambda_{t(F, H)}\right) \chi\left(H, \Lambda_{H}\right),
$$

where

$$
U_{\ell}:=\left\{(F, H) \mid F \in \mathcal{T}_{\ell}, 0 \neq H \supsetneqq F\right\} .
$$

Note that $U_{\ell}$ is the disjoint union of the sets

$$
U_{\ell, \ell}:=\left\{(F, H) \in U_{\ell} \mid j(H)=\ell\right\} \text { and } U_{\geq \ell+1}:=\left\{(F, H) \in U_{\ell} \mid j(H) \geq \ell+1\right\} .
$$

On the one hand, for $(F, H) \in U_{\ell, \ell}$, we have $J(F) \subset J(H)$. The fact that they have the same cardinal implies the equality $J(F)=J(H)$. Moreover, $F=C_{J(F)}$. Since $H \supsetneqq F$, we have $H \in \mathcal{P}_{\ell}^{\prime}$ and obtain

$$
\sum_{(F, H) \in U_{\ell, \ell}} \lambda_{F} \phi\left(t(F, H), \Lambda_{t(F, H)}\right) \chi\left(H, \Lambda_{H}\right)=\sum_{H \in \mathcal{P}_{\ell}^{\prime}} \sum_{F \in \mathcal{T}_{\ell}(H)} \lambda_{F} \phi\left(t(F, H), \Lambda_{t(F, H)}\right) \chi\left(H, \Lambda_{H}\right) .
$$


On the other hand,

$$
\begin{aligned}
\sum_{(F, H) \in U_{\ell, \geq \ell+1}} \lambda_{F} \phi\left(t(F, H), \Lambda_{t(F, H)}\right) \chi\left(H, \Lambda_{H}\right) & =\sum_{0 \neq H, j(H) \geq \ell+1} \sum_{F \in \mathcal{T}_{\ell}(H)} \lambda_{F} \phi\left(t(F, H), \Lambda_{t(F, H)}\right) \chi\left(H, \Lambda_{H}\right) \\
& =\sum_{k \geq \ell+1} \sum_{H \in \mathcal{P}_{k}} \sum_{F \in \mathcal{T}_{\ell}(H)} \lambda_{F} \phi\left(t(F, H), \Lambda_{t(F, H)}\right) \chi\left(H, \Lambda_{H}\right) .
\end{aligned}
$$

Inserting the last two identities into Eq. (33) yields the following expression for the left hand side of Eq. (30)

$$
\begin{aligned}
& \sum_{F \in \mathcal{T}_{\ell} \amalg}\left(\phi(c(F))-\sum_{m=2}^{\ell-1} \sum_{H \in \mathcal{T}_{m}^{\prime}(F)} \lambda_{H} \phi\left(t(H, F), \Lambda_{t(H, F)}\right)\right) \chi\left(F, \Lambda_{F}\right) \\
= & \sum_{F \in \mathcal{P}_{\ell}^{\prime}}\left(\phi(c(F))-\sum_{m=2}^{\ell-1} \sum_{H \in \mathcal{T}_{m}(F)} \lambda_{H} \phi\left(t(H, F), \Lambda_{t(H, F)}\right)\right) \chi\left(F, \Lambda_{F}\right) \\
& -\sum_{G \in \mathcal{P}_{C, 0}} \sum_{F \in \alpha^{-1}(G)_{\ell} \cap \mathcal{T}} \phi\left(t(C, G), \Lambda_{t(C, G)}\right) \chi(F)+\sum_{F \in \mathcal{T}_{\ell}} \lambda_{F} \chi\left(F, \Lambda_{F}\right) \\
= & \sum_{F \in \mathcal{P}_{\ell}^{\prime}}\left(\phi(c(F))-\sum_{m=2}^{\ell} \sum_{H \in \mathcal{T}_{m}(F)} \lambda_{H} \phi\left(t(H, F), \Lambda_{t(H, F)}\right)\right) \chi\left(F, \Lambda_{F}\right) \\
& -\sum_{G \in \mathcal{P}_{C, 0}} \sum_{F \in \alpha^{-1}(G)_{\ell} \cap \mathcal{T}} \phi\left(t(C, G), \Lambda_{t(C, G)}\right) \chi\left(F, \Lambda_{F}\right) \\
& +(\psi-\phi)\left(\sum_{F \in \mathcal{T}_{\ell}} \lambda_{F}\left(F, \Lambda_{F}\right)\right) \\
& -\sum_{k \geq \ell+1} \sum_{H \in \mathcal{P}_{k}} \sum_{F \in \mathcal{T}_{\ell}(H)} \lambda_{F} \phi\left(t(F, H), \Lambda_{t(H, F)}\right) \chi\left(H, \Lambda_{H}\right) .
\end{aligned}
$$

By Eq. (27), the cofactor of $\chi\left(F, \Lambda_{F}\right)$ in the first sum in the above formula is

$$
\begin{gathered}
\phi(c(F))-\sum_{m=2}^{\ell} \sum_{H \in \mathcal{T}_{m}(F)} \lambda_{H} \phi\left(t(H, F), \Lambda_{t(H, F)}\right) \\
= \begin{cases}0, & \text { for } F \in \mathcal{P}_{C, \geq 2}^{\prime}, \\
-\phi\left(t(C, F), \Lambda_{t(C, F)}\right), & \text { for } F \in \mathcal{P}_{S \mathrm{I}}^{\prime}, \\
-\phi\left(t(C, F), \Lambda_{t(C, F)}\right)=0, & \text { for } F \in \mathcal{P}_{N}^{\prime},\end{cases}
\end{gathered}
$$

where we have applied the notations in Eq. (32), and in the last case, $\phi\left(t(C, F), \Lambda_{t(C, F)}\right)=0$ using Lemma 3.10.(C). Thus this sum becomes

$$
\begin{aligned}
-\sum_{H \in \mathcal{P}_{\mathrm{SI}}^{\prime}} \phi\left(t(C, H), \Lambda_{t(C, H)}\right) \chi\left(H, \Lambda_{H}\right) & =-\sum_{H \in \mathcal{P}_{\mathrm{SI}}^{\prime}} \phi\left(t(C, \alpha(H)), \Lambda_{t(C, \alpha(H))}\right) \chi\left(H, \Lambda_{H}\right) \\
& =-\sum_{G \in \mathcal{P}_{C, 0}} \sum_{H \in \alpha^{-1}(G) \epsilon_{\ell} \cap \mathcal{P}^{\prime}} \phi\left(t(C, G), \Lambda_{t(C, G)}\right) \chi\left(H, \Lambda_{H}\right) .
\end{aligned}
$$

This proves that the left hand side of Eq. (30) agrees with the right hand side. 
Proof of Eq. (31): We prove the equation by induction on $\ell \geq 2$. We first verify the case when $\ell=2$. By definition,

$$
\begin{aligned}
\chi\left(\left(C, \Lambda_{C}\right)-\sum_{i}\left(C_{i}, \Lambda_{C_{i}}\right)\right)= & (\psi-\phi)\left(\left(C, \Lambda_{C}\right)-\sum_{i}\left(C_{i}, \Lambda_{C_{i}}\right)\right)-\sum_{F \in \mathcal{P}} \phi(c(F)) \chi\left(F, \Lambda_{F}\right) \\
= & (\psi-\phi)\left(\left(C, \Lambda_{C}\right)-\sum_{i}\left(C_{i}, \Lambda_{C_{i}}\right)\right) \\
& -\sum_{F \in \mathcal{P}_{0} \cup \mathcal{P}_{1}} \phi(c(F)) \chi\left(F, \Lambda_{F}\right)-\sum_{k \geq 2} \sum_{F \in \mathcal{P}_{k}} \phi(c(F)) \chi\left(F, \Lambda_{F}\right) .
\end{aligned}
$$

Now we see that the first and third sums on the right hand side readily agree with the corresponding sums on the right hand side of Eq. (31).

For the second sum, note that

$$
\mathcal{P}_{0}=\mathcal{P}_{C, 0}, \quad \mathcal{P}_{1}=\mathcal{P}_{C, 1} \coprod \mathcal{P}_{C, \geq 2,1} \coprod \mathcal{P}_{\mathrm{SI}, 1} \bigsqcup \mathcal{P}_{N, 1}
$$

By Lemma 3.10.(国), $c(F)=0$ for $F \in \mathcal{P}_{C, 1}$. Also $\mathcal{P}_{C, \geq 2,1}=\emptyset$ by definition. By Lemma 3.10.(河), we have $\phi(t(C, F))=0$ for $F \in \mathcal{P}_{N, 1}$.

Notice that for $G \in \mathcal{P}_{1}$, if $F^{G} \in \alpha^{-1}(G)$ and $j\left(F^{G}\right)=1$, then we can take $J\left(F^{G}\right)=\{i\}$. So by Lemma 3.10, $t(C, F)=t\left(C_{i}, F^{G}\right)$. This proves that the second sum agrees with the second sum in Eq. (31) when $\ell=2$. Therefore Eq. (31) holds when $\ell=2$.

The inductive step follows from Eq. (30) applied to the third sum.

Now we are ready to prove Theorem 3.13 .

Proof. (of Theorem 3.13) We prove the statement by induction on the dimension of $C$, the case $\operatorname{dim} C=1$ being trivial. Assume that the theorem holds for cones of dimension less or equal to $k \geq 1$ and consider a cone $C$ of dimension $k+1$. Let a subdivision of $C$ be given. Taking $\ell$ sufficiently large (say greater than the number $n$ of the $C_{i}$ 's in the subdivision of $C$ ) in Eq. (31), we have

$$
\begin{aligned}
\chi\left(\left(C, \Lambda_{C}\right)-\sum_{i}\left(C_{i}, \Lambda_{C_{i}}\right)\right)= & (\psi-\phi)\left(\left(C, \Lambda_{C}\right)-\sum_{i}\left(C_{i}, \Lambda_{C_{i}}\right)-\sum_{F \in \mathcal{T}_{\geq 2}} \lambda_{F}\left(F, \Lambda_{F}\right)\right) \\
& -\sum_{G \in \mathcal{P}_{C, 0}} \phi\left(t(C, G), \Lambda_{t(C, G)}\right)\left(\chi\left(G, \Lambda_{G}\right)-\sum_{H \in \alpha^{-1}(G)} \chi\left(H, \Lambda_{H}\right)\right) .
\end{aligned}
$$

By the discrete closed subdivision property of $\phi$ and $\psi$, the first term on the right hand side is zero. By the induction hypothesis, the second term is also zero since $\left\{H \in \alpha^{-1}(G)\right\}$ gives a subdivision of $G$ by Lemma 3.7.(B). This completes the induction.

\section{EULER-MaClaurin FORMULAE FOR LATTICE CONES}

We derive the Euler-Maclaurin formula from the above results combined with an Algebraic Birkhoff Factorization on lattice cones, which generalizes Connes-Kreimer renormalization scheme. 
4.1. Meromorphicity of generating functions. From now on, we work in the filtered lattice space $\mathbb{R}^{\infty}$, with the standard lattice $\Lambda_{\infty}=\mathbb{Z}^{\infty}$ and a fixed basis $\left\{e_{1}, e_{2}, \cdots\right\}$.

To a cone $C$ in a lattice filtered space $V$, one can assign two meromorphic functions: the generating function (or the exponential discrete sum) $S(C)$ and the exponential integral $I_{V}(C)$ [1], 7, 10, 13]. These can be extended to a lattice cone by the subdivision technique.

It is simple for simplicial cones. If $\left(C, \Lambda_{C}\right) \in \mathfrak{C}_{k}$ is a simplicial lattice cone (so in particular it is strongly convex), then the set

$$
\check{C}^{-}:=\check{C}_{k}^{-}:=\left\{\vec{\varepsilon}:=\sum_{i=1}^{k} \varepsilon_{i} e_{i}^{*} \mid\langle\vec{x}, \vec{\varepsilon}\rangle<0 \text { for all } \vec{x} \in C\right\}
$$

is of dimension $k$. Here $\langle\vec{x}, \vec{\varepsilon}\rangle$ denotes the natural pairing $V_{k} \otimes V_{k}^{*} \rightarrow \mathbb{R}$. Let $C^{o}$ denote the interior of $C$. For $\vec{\varepsilon} \in \check{C}^{-}$, then define

$$
S^{o}\left(C, \Lambda_{C}\right)(\vec{\varepsilon}):=\sum_{\vec{n} \in C^{o} \cap \Lambda_{C}} e^{\langle\vec{n}, \vec{\varepsilon}\rangle}
$$

If $v_{1}, \cdots v_{k} \in \Lambda_{C}$ is a set of primary generators of $C$, and $u_{1}, \cdots, u_{k}$ is a basis of $\Lambda_{C}$, for $1 \leq i \leq k$, let $v_{i}=\sum_{j=1}^{k} a_{j i} u_{j}, a_{j i} \in \mathbb{Z}$. Define linear functions $L_{i}:=L_{v_{i}}:=\sum_{j=1}^{k} a_{j i}\left\langle u_{j}, \vec{\varepsilon}\right\rangle$ and let $w\left(C, \Lambda_{C}\right)$ denote the absolute value of the determinant of the matrix $\left[a_{i j}\right]$, then

$$
I\left(C, \Lambda_{C}\right)(\vec{\varepsilon}):=(-1)^{k} \frac{w\left(C, \Lambda_{C}\right)}{L_{1} \cdots L_{k}} .
$$

Remark 4.1. We use a sign convention that is different from [10] in order to make the EulerMaclaurin formula simpler.

Then by the subdivision technique, we have

Proposition-Definition 4.2. For a lattice cone $\left(C, \Lambda_{C}\right)$, the germ of functions $\sum_{F \in \mathcal{F}^{o}(\underline{C})} S^{o}\left(F, \Lambda_{F}\right)$ and $\sum_{i \in[n]} I\left(C_{i}, \Lambda_{C_{i}}\right)$ do not depend on the choice of the simplicial subdivision $\underline{C}=\left\{\left(C_{i}, \Lambda_{C_{i}}\right)\right\}_{i \in[n]}$ of $\left(C, \Lambda_{C}\right)$. Thus we define

$$
S^{o}\left(C, \Lambda_{C}\right):=\sum_{F \in \mathcal{F}^{o}(\underline{C})} S^{o}\left(F, \Lambda_{F}\right)
$$

and

$$
I\left(C, \Lambda_{C}\right)=\sum_{i \in[n]} I\left(C_{i}, \Lambda_{C_{i}}\right)
$$

for any simplicial subdivision $\underline{C}=\left\{\left(C_{i}, \Lambda_{C_{i}}\right)\right\}_{i \in[n]}$ of $\left(C, \Lambda_{C}\right)$.

We next view the generating functions $S^{o}\left(C, \Lambda_{C}\right)(\vec{\varepsilon})$ as meromorphic germs with linear poles at zero, see [11] for a more detailed discussion.

Definition 4.3. Let $k$ be a positive integer.

(a) A germ of meromorphic functions at $\mathbf{0}$ on $\mathbb{C}^{k}$ is the quotient of two holomorphic functions in a neighborhood of 0 inside $\mathbb{C}^{k}$.

(b) A germ of meromorphic functions $f(\vec{\varepsilon})$ on $\mathbb{C}^{k}$ is said to have linear poles at zero with lattice coefficients if there exist vectors $L_{1}, \cdots, L_{n} \in \Lambda_{k} \otimes \mathbb{Q}$ (possibly with repetitions) such that $f \prod_{i=1}^{n} L_{i}$ is a holomorphic germ at zero whose Taylor expansion has lattice coefficients. 
(c) We will denote by $\mathcal{M}_{\mathbb{Q}}\left(\mathbb{C}^{k}\right)$ the set of germs of meromorphic functions on $\mathbb{C}^{k}$ with linear poles at zero with lattice coefficients. It is a linear subspace over $\mathbb{Q}$.

Then composing with the projection $\mathbb{C}^{k+1} \rightarrow \mathbb{C}^{k}$ dual to the inclusion $j_{k}: \mathbb{C}^{k} \rightarrow \mathbb{C}^{k+1}$ yields the embedding

$$
\mathcal{M}_{\mathbb{Q}}\left(\mathbb{C}^{k}\right) \hookrightarrow \mathcal{M}_{\mathbb{Q}}\left(\mathbb{C}^{k+1}\right)
$$

thus giving rise to the direct limit

$$
\mathcal{M}_{\mathbb{Q}}\left(\mathbb{C}^{\infty}\right):=\underset{\lim }{\longrightarrow} \mathcal{M}_{\mathbb{Q}}\left(\mathbb{C}^{k}\right)=\bigcup_{k=1}^{\infty} \mathcal{M}_{\mathbb{Q}}\left(\mathbb{C}^{k}\right) .
$$

Lemma 4.4. For a simplicial lattice cone $\left(C, \Lambda_{C}\right) \in \mathfrak{C}_{k}$, the germs of functions $S^{o}\left(C, \Lambda_{C}\right)(\vec{\varepsilon})$ lies in $\mathcal{M}_{\mathbb{Q}}\left(\mathbb{C}^{k}\right)$.

Proof. We first prove the proposition for a smooth lattice cone $\left(C, \Lambda_{C}\right)$. Let $C=\left\langle v_{1}, \cdots, v_{m}\right\rangle$ with $\left\{v_{1}, \cdots, v_{m}\right\}$ being a basis of $\Lambda_{C}$. Since an element $\vec{x}$ in $C \cap \Lambda_{C}$ can be written in a unique way as $\sum_{j=1}^{m} n_{j} v_{j}$ where $n_{j} \in \mathbb{Z}_{\geq 0}$, for $\vec{\varepsilon}=\sum_{j=1}^{m} \varepsilon_{j} e_{j}^{*} \in \check{C}^{-}$, we have

$$
S^{o}\left(C, \Lambda_{C}\right)(\vec{\varepsilon}):=\prod_{j=1}^{m} \sum_{n_{j} \in \mathbb{Z}_{\geq 1}} e^{n_{j}\left\langle v_{j}, \vec{\varepsilon}\right\rangle}=\prod_{j=1}^{m} \frac{e^{\left\langle v_{j}, \vec{\varepsilon}\right\rangle}}{1-e^{\left\langle v_{j}, \vec{\varepsilon}\right\rangle}}=\prod_{j=1}^{m} \frac{e^{L_{j}(\vec{\varepsilon})}}{1-e^{L_{j}(\vec{\varepsilon})}},
$$

where $L_{j}(\vec{\varepsilon})=\left\langle v_{j}, \vec{\varepsilon}\right\rangle$. They are holomorphic on $\check{C}^{-}$and extend to germs of meromorphic functions on $\mathbb{C}^{k}$ with simple linear poles at $L_{1}(\vec{\varepsilon})=0, \cdots, L_{n}(\vec{\varepsilon})=0$.

Indeed, from the generating power series $\frac{x}{e^{x}-1}=\sum_{n=0}^{\infty} B_{n} \frac{x^{n}}{n !}$ of Bernoulli numbers, we have that $\frac{1}{1-e^{x}}=-\frac{1}{x} \frac{x}{e^{x}-1}$ is in $\mathcal{M}_{\mathbb{Q}}(\mathbb{C})$. Then the same holds for $\frac{e^{x}}{1-e^{x}}=\frac{1}{1-e^{x}}-1$. Thus for each linear form $L$ on $\mathbb{C}^{k}$ with lattice coefficients, both $\frac{L}{1-e^{L}}$ and $\frac{e^{L}}{1-e^{L}}$ are in $\mathcal{M}_{\mathbb{Q}}\left(\mathbb{C}^{k}\right)$. For a smooth lattice cone, the conclusion that $S^{o}\left(C, \Lambda_{C}\right)(\vec{\varepsilon})$ lies in $\mathcal{M}_{\mathbb{Q}}\left(\mathbb{C}^{k}\right)$ follows from Eq. (36) since $\mathcal{M}_{\mathbb{Q}}\left(\mathbb{C}^{k}\right)$ is closed under multiplication.

Next for a simplicial lattice cone $\left(C, \Lambda_{C}\right)$, we prove the statement by taking a smooth subdivision and applying Proposition-Definition 4.2, noting that faces of a smooth lattice cone are smooth by Proposition 2.12.

Therefore, we have linear map

$$
S^{o}: \mathbb{Q} \mathfrak{C} \rightarrow \mathcal{M}_{\mathbb{Q}}\left(\mathbb{C}^{\infty}\right), \quad\left(C, \Lambda_{C}\right) \mapsto S^{o}\left(C, \Lambda_{C}\right) .
$$

By definition, the following conclusion holds.

Corollary 4.5. Let $\left(C, \Lambda_{C}\right)$ be a lattice cone and let $\underline{C}=\left\{\left(C_{1}, \Lambda_{C}\right), \cdots,\left(C_{r}, \Lambda_{C}\right)\right\}$ be a (not necessarily simplicial) subdivision of $C$. Then we have

$$
S^{o}\left(C, \Lambda_{C}\right)=\sum_{F \in \mathcal{F}^{o}(\underline{C})} S^{o}\left(F, \Lambda_{F}\right)
$$

and

$$
I\left(C, \Lambda_{C}\right)=\sum_{i \in[n]} I\left(C_{i}, \Lambda_{C_{i}}\right)
$$

in $\mathcal{M}_{\mathbb{Q}}\left(\mathbb{C}^{\infty}\right)$, that is, $S^{o}$ has the discrete open subdivision property and I has the continuous subdivision property. 
Definition 4.6. For a lattice cone $\left(C, \Lambda_{C}\right) \in \mathfrak{C}_{k}$, define its (closed) generating function by

$$
S^{c}\left(C, \Lambda_{C}\right)=\sum_{F \leq C} S^{o}\left(F, \Lambda_{F}\right),
$$

giving rise to the linear map

$$
S^{c}: \mathbb{Q}\left(\mathfrak{L} \rightarrow \mathcal{M}_{\mathbb{Q}}\left(\mathbb{C}^{\infty}\right), \quad\left(C, \Lambda_{C}\right) \mapsto S^{c}\left(C, \Lambda_{C}\right) .\right.
$$

By Proposition 3.12, we have

Corollary 4.7. $S^{c}: \mathbb{Q} \mathfrak{C} \rightarrow \mathcal{M}_{\mathbb{Q}}\left(\mathbb{C}^{\infty}\right)$ has the discrete closed subdivision property.

We now state one more key property of $S^{o}\left(C, \Lambda_{C}\right)$ and $S^{c}\left(C, \Lambda_{C}\right)$.

Proposition 4.8. If $\left(C, \Lambda_{C}\right)$ is not strictly convex, then $S^{o}\left(C, \Lambda_{C}\right)$ and $S^{c}\left(C, \Lambda_{C}\right)$ are both zero.

Proof. First consider the case when $C$ is a one-dimensional subspace. So $\left(C, \Lambda_{C}\right)=\left(\mathbb{R}_{\geq 0} u, \mathbb{Z} u\right)$. Then $\{\langle u\rangle,\langle-u\rangle\}$ is a smooth subdivision of $C$. Then as in Eq. (36), we obtain

$S^{o}(C, \mathbb{Z} u)(\vec{\varepsilon})=S^{o}(\langle u\rangle, \mathbb{Z} u)(\vec{\varepsilon})+S^{o}(\{0\},\{0\})(\vec{\varepsilon})+S^{o}(\langle-u\rangle, \mathbb{Z} u)(\vec{\varepsilon})=\frac{e^{\langle u, \vec{\varepsilon}\rangle}}{1-e^{<u, \vec{\varepsilon}\rangle}}+1+\frac{e^{<-u, \vec{\varepsilon}\rangle}}{1-e^{<-u, \vec{\varepsilon}\rangle}}=0$.

Since $C=\mathbb{R} u$ does not have a proper face, by Eq. (37) we have

$$
S^{c}(C, \mathbb{Z} u)(\vec{\varepsilon})=S^{o}(C, \mathbb{Z} u)(\vec{\varepsilon})=0 .
$$

Next consider the case $\left(C, \Lambda_{C}\right)$ where $C$ is a linear space of dimension $k$. Then $C$ has no proper face. Take a lattice basis $\left\{v_{1}, \cdots, v_{k}\right\}$ of $\Lambda_{C}$ and denote $C_{\alpha_{1} \alpha_{2} \cdots \alpha_{k}}:=\left\langle\alpha_{1} v_{1}, \alpha_{2} v_{2}, \cdots, \alpha_{k} v_{k}\right\rangle$ for $\alpha_{i} \in \mathbb{R}, 1 \leq i \leq k$. Then the family of lattice cones $\left\{\left(C_{\alpha_{1} \alpha_{2} \cdots \alpha_{k}}, \Lambda_{C}\right) \mid \alpha_{i}= \pm 1,1 \leq i \leq k\right\}$ provides a simplicial subdivision of $\left(C, \Lambda_{C}\right)$. Thus $\mathcal{F}^{o}\left(C \sim \cup C_{\alpha_{1} \alpha_{2} \cdots \alpha_{k}}\right)=\left\{C_{\alpha_{1} \alpha_{2} \cdots \alpha_{k}} \mid \alpha_{i}=0, \pm 1,1 \leq i \leq k\right\}$ and

$$
S^{o}\left(C_{\alpha_{1} \alpha_{2} \cdots \alpha_{k}}, \Lambda_{C} \cap \operatorname{lin}\left(C_{\alpha_{1} \alpha_{2} \cdots \alpha_{k}}\right)\right)(\vec{\varepsilon})=\prod_{i, \alpha_{i} \neq 0} \frac{e^{<\alpha_{i} v_{i}, \vec{\varepsilon}>}}{1-e^{<\alpha_{i} v_{i}, \vec{\varepsilon}>}} .
$$

Thus

$$
\begin{aligned}
S^{o}\left(C, \Lambda_{C}\right)(\vec{\varepsilon}) & =\sum_{\alpha_{i}=0, \pm 1,1 \leq i \leq k} S^{o}\left(C_{\alpha_{1} \alpha_{2} \cdots \alpha_{k}}, \Lambda_{C} \cap \operatorname{lin}\left(C_{\alpha_{1} \alpha_{2} \cdots \alpha_{k}}\right)\right)(\vec{\varepsilon}) \\
& =\prod_{i}\left(\frac{e^{<v_{i}, \vec{\varepsilon}>}}{1-e^{<v_{i}, \vec{\varepsilon}>}}+1+\frac{e^{<-v_{i}, \vec{\varepsilon}>}}{1-e^{<-v_{i}, \vec{\varepsilon}>}}\right)=0 .
\end{aligned}
$$

Finally consider the case when $C$ is a cone that contains a linear subspace. By Proposition 3.4.(a) in [10], we have $C=\left\{v+u \mid v \in L, u \in C^{\prime}\right\}$, where $L$ is a linear subspace and $C^{\prime}$ is a strongly convex cone in the orthogonal complement $\operatorname{lin}(L ; \operatorname{lin}(C))^{\perp}$ of $L$ in $\operatorname{lin}(C)$. Therefore any element in $C$ has a unique decomposition $v+u$ with $v \in L$ and $u \in C^{\prime}$. Let $\Lambda_{L}$ and $\Lambda_{C^{\prime}}$ be the projection of $\Lambda_{C}$ in $L$ and $\operatorname{lin}^{\perp}(L ; \operatorname{lin}(C))$ respectively. Picking a basis $\left\{v_{1}, \cdots, v_{k}\right\}$ of $\Lambda_{L}$, the set $\left\{C_{\alpha_{1}, \alpha_{2}, \cdots, \alpha_{k}}+C^{\prime} \mid \alpha_{i}= \pm 1\right\}$ provides a subdivision of $C$. Further,

$$
S^{o}\left(C_{\alpha_{1}, \alpha_{2}, \cdots, \alpha_{k}}+C^{\prime}, \Lambda_{C}\right)(\vec{\varepsilon})=S^{o}\left(C_{\alpha_{1}, \alpha_{2}, \cdots, \alpha_{k}}, \Lambda_{L}\right)(\vec{\varepsilon}) S^{o}\left(C^{\prime}, \Lambda_{C^{\prime}}\right)(\vec{\varepsilon}) .
$$

So as in the case of a linear subspace, we have

$$
S^{o}\left(C, \Lambda_{C}\right)(\vec{\varepsilon})=S^{o}\left(L, \Lambda_{L}\right)(\vec{\varepsilon}) S^{o}\left(C^{\prime}, \Lambda_{C^{\prime}}\right)(\vec{\varepsilon})=0 .
$$

For $S^{c}\left(C, \Lambda_{C}\right)$, note that any face of $C$ contains the above $L$. Therefore $S^{c}\left(C, \Lambda_{C}\right)=0$ by Eq. (37). 
4.2. Algebraic Birkhoff Factorization. We first give a general formulation of the Algebraic Birkhoff Factorization before applying it to the study of lattice cones.

4.2.1. The general result. We give a generalization of the Algebraic Birkhoff Factorization of Connes-Kreimer [5] for connected coalgebras without the need for either a Hopf algebra or a Rota-Baxter algebra. We begin with a lemma (see e.g. [16, Prop. II.3.1]).

Lemma 4.9. Let $\mathbf{C}=\bigoplus_{n \geq 0} \mathbf{C}^{(n)}$ be a connected cograded coaugmented coalgebra with coaumentation $u$. Denote $J=u(1)$ and let $A$ be a commutative algebra with unit $1_{A}$. Let $*$ be the convolution product on the algebra $\mathcal{L}(\mathbf{C}, A)$ of linear maps from $\mathbf{C}$ to $A$ and let $\varphi \in \mathcal{L}(\mathbf{C}, A)$ be such that $\varphi(J)=1_{A}$. Then $\varphi$ has a convolution inverse $\varphi^{*(-1)}: \mathbf{C} \rightarrow$ A for which $\varphi^{*(-1)}(J)=1_{A}$. Consequently,

$$
\mathcal{G}(\mathbf{C}, A):=\left\{\varphi \in \mathcal{L}(\mathbf{C}, A) \mid \varphi(J)=1_{A}\right\}
$$

endowed with the convolution product is a group.

Theorem 4.10. Let $\mathbf{C}=\bigoplus_{n \geq 0} \mathbf{C}^{(n)}$ be a connected cograded coaugmented coalgebra. Let $A$ be a unitary algebra. Let $A=A_{1} \oplus A_{2}$ be a linear decomposition such that $1_{A} \in A_{1}$. Let $P$ be the projection of $A$ to $A_{1}$ along $A_{2}$. Given $\varphi \in \mathcal{G}(\mathbf{C}, A)$, define maps $\varphi_{i} \in \mathcal{G}(\mathbf{C}, A), i=1,2$, by the following recursive formulae on $\operatorname{ker} \varepsilon$ :

$$
\begin{aligned}
& \varphi_{1}(x)=-P\left(\varphi(x)+\sum_{(x)} \varphi_{1}\left(x^{\prime}\right) \varphi\left(x^{\prime \prime}\right)\right) \\
& \varphi_{2}(x)=\left(\operatorname{id}_{A}-P\right)\left(\varphi(x)+\sum_{(x)} \varphi_{1}\left(x^{\prime}\right) \varphi\left(x^{\prime \prime}\right)\right) .
\end{aligned}
$$

(a) We have $\varphi_{i}(\operatorname{ker} \varepsilon) \subseteq A_{i}$ (hence $\left.\varphi_{i}: \mathbf{C} \rightarrow \mathbf{k} 1_{A}+A_{i}\right)$. Moreover, the following factorization holds

$$
\varphi=\varphi_{1}^{*(-1)} * \varphi_{2}
$$

(b) $\varphi_{1}$ and $\varphi_{2}$ are the unique maps in $\mathcal{G}(\mathbf{C}, A)$ such that $\varphi_{i}(\operatorname{ker} \varepsilon) \subseteq A_{i}$ for $i=1,2$, and $E q$. (40) holds.

(c) If moreover $A_{1}$ is a subalgebra of $A$, then $\phi_{1}^{*(-1)}$ lies in $\mathcal{G}\left(\mathbf{C}, A_{1}\right)$.

Proof. (国) The inclusion $\varphi_{i}(\operatorname{ker} \varepsilon) \subseteq A_{i}, i=1,2$, follows from the definitions. Further

$$
\varphi_{2}(x)=\left(\operatorname{id}_{A}-P\right)\left(\varphi(x)+\sum_{(x)} \varphi_{1}\left(x^{\prime}\right) \varphi\left(x^{\prime \prime}\right)\right)=\varphi(x)+\varphi_{1}(x)+\sum_{(x)} \varphi_{1}\left(x^{\prime}\right) \varphi\left(x^{\prime \prime}\right)=\left(\varphi_{1} * \varphi\right)(x) .
$$

Since $\varphi_{1}(J)=1_{A}, \varphi_{1}$ is invertible for the convolution product in $A$ by Lemma 4.9. Then Eq. (40) follows.

(D) Suppose there are $\psi_{i} \in \mathcal{G}(\mathbf{C}, A), i=1,2$, with $\psi_{i}(\operatorname{ker} \varepsilon) \subseteq A_{i}$ such that $\varphi=\psi_{1}^{*(-1)} * \psi_{2}$. We prove $\varphi_{i}(x)=\psi_{i}(x)$ for $i=1,2, x \in \mathbf{C}^{(k)}$ by induction on $k \geq 0$. These equations hold for $k=0$. Assume that the equations hold for $x \in \mathbf{C}^{(k)}$ where $k \geq 0$. For $x \in \mathbf{C}^{(k+1)} \subseteq \operatorname{ker}(\varepsilon)$, by $\varphi_{2}=\varphi_{1} * \varphi$ and $\psi_{2}=\psi_{1} * \varphi$, we have

$$
\varphi_{2}(x)=\varphi_{1}(x)+\varphi(x)+\sum_{(x)} \varphi_{1}\left(x^{\prime}\right) \varphi\left(x^{\prime \prime}\right), \quad \psi_{2}(x)=\psi_{1}(x)+\varphi(x)+\sum_{(x)} \psi_{1}\left(x^{\prime}\right) \varphi\left(x^{\prime \prime}\right),
$$


where we have made use of $\varphi_{1}(J)=\psi_{1}(J)=\varphi(J)=1_{A}$. Hence by the induction hypothesis, we have

$$
\varphi_{2}(x)-\psi_{2}(x)=\varphi_{1}(x)-\psi_{1}(x)+\sum_{(x)}\left(\varphi_{1}\left(x^{\prime}\right)-\psi_{1}\left(x^{\prime}\right)\right) \varphi\left(x^{\prime \prime}\right)=\varphi_{1}(x)-\psi_{1}(x) \in A_{1} \cap A_{2}=\{0\} .
$$

Thus $\varphi_{i}(x)=\psi_{i}(x), i=1,2$, for all $x \in \operatorname{ker}(\varepsilon)$.

() If $A_{1}$ is a subalgebra, then it follows from Lemma 4.9 applied to $A_{1}$ instead of $A$, that $\varphi_{1}$ is invertible in $A_{1}$.

4.2.2. Application to lattice cones. We now focus on the filtered lattice space $\mathbb{R}^{\infty}$, let $Q(\cdot, \cdot)$ denote the inner product chosen in Eq. (2). In this setup, we have constructed two linear maps:

$$
S^{o}: \mathbb{Q} \mathfrak{C} \rightarrow \mathcal{M}_{\mathbb{Q}}\left(\mathbb{C}^{\infty}\right) \text { and } S^{c}: \mathbb{Q} \mathbb{C} \rightarrow \mathcal{M}_{\mathbb{Q}}\left(\mathbb{C}^{\infty}\right) \text {. }
$$

Let $\mathcal{M}_{\mathbb{Q},+}\left(\mathbb{C}^{k}\right)$ denote the space of germs of holomorphic functions at zero in $\mathbb{C}^{k}$ whose Taylor expansions at zero have lattice coefficients. We set

$$
\mathcal{M}_{\mathbb{Q},+}\left(\mathbb{C}^{\infty}\right):=\lim _{\longrightarrow} \mathcal{M}_{\mathbb{Q},+}\left(\mathbb{C}^{k}\right)=\bigcup_{k=0}^{\infty} \mathcal{M}_{\mathbb{Q},+}\left(\mathbb{C}^{k}\right)
$$

Then $\mathcal{M}_{\mathbb{Q},+}\left(\mathbb{C}^{k}\right)\left(\operatorname{resp} . \mathcal{M}_{\mathbb{Q},+}\left(\mathbb{C}^{\infty}\right)\right)$ is a unitary subalgebra of $\mathcal{M}_{\mathbb{Q}}\left(\mathbb{C}^{k}\right)\left(\operatorname{resp} . \mathcal{M}_{\mathbb{Q}}\left(\mathbb{C}^{\infty}\right)\right)$.

The filtered lattice Euclidean space $\left(\mathbb{R}^{\infty}, Q(\cdot, \cdot)\right)$ allows us to apply [11, Theorem 4.4] to obtain the linear decomposition

$$
\mathcal{M}_{\mathbb{Q}}\left(\mathbb{C}^{\infty}\right)=\mathcal{M}_{\mathbb{Q},+}\left(\mathbb{C}^{\infty}\right) \oplus \mathcal{M}_{\mathbb{Q},-}\left(\mathbb{C}^{\infty}\right) .
$$

Here $\mathcal{M}_{\mathbb{Q},-}\left(\mathbb{C}^{\infty}\right)$ is the subspace spanned by polar germs, defined to be germs of meromorphic functions at zero of the form

$$
\frac{h\left(\ell_{1}, \cdots, \ell_{m}\right)}{L_{1}^{s_{1}} \cdots L_{n}^{S_{n}}}
$$

where

(a) $h$ lies in $\mathcal{M}_{\mathbb{Q},+}\left(\mathbb{C}^{m}\right)$,

(b) $\ell_{1}, \cdots, \ell_{m}, L_{1}, \cdots, L_{n}$ lie in $\Lambda_{k} \otimes \mathbb{Q}$, with $L_{1}, \cdots, L_{n}$ linearly independent, such that

$$
Q\left(\ell_{i}, L_{j}\right)=0 \quad \text { for all }(i, j) \in[m] \times[n] .
$$

(c) $s_{1}, \cdots, s_{n}$ are positive integers.

Since $\mathcal{M}_{\mathbb{Q},+}\left(\mathbb{C}^{\infty}\right)$ is a unitary subalgebra, the Algebraic Birkhoff Factorization in Theorem 4.10 applies, with $\mathbf{C}=\mathbb{Q C}$ and

$$
A=\mathcal{M}_{\mathbb{Q}}\left(\mathbb{C}^{\infty}\right), \quad A_{1}=\mathcal{M}_{\mathbb{Q},+}\left(\mathbb{C}^{\infty}\right), \quad A_{2}=\mathcal{M}_{\mathbb{Q},-}\left(\mathbb{C}^{\infty}\right), \quad P=\pi_{+}: \mathcal{M}_{\mathbb{Q}}\left(\mathbb{C}^{\infty}\right) \rightarrow \mathcal{M}_{\mathbb{Q},+}\left(\mathbb{C}^{\infty}\right),
$$

which is the orthogonal projection onto the holomorphic part $\mathcal{M}_{\mathbb{Q},+}\left(\mathbb{C}^{\infty}\right)$ along the space $\mathcal{M}_{\mathbb{Q},-}\left(\mathbb{C}^{\infty}\right)$ of polar germs by means of the decomposition in Eq. (41). We consequently obtain the following theorem.

Corollary 4.11. (Algebraic Birkhoff Factorization for generating functions) For the linear map

$$
S^{\#}: \mathbb{Q} C \rightarrow \mathcal{M}_{\mathbb{Q}}\left(\mathbb{C}^{\infty}\right),
$$

where \# $\in\{o, c\}$, there exist unique linear maps $S_{1}^{\#}: \mathbb{Q} \mathfrak{C} \rightarrow \mathcal{M}_{\mathbb{Q},+}\left(\mathbb{C}^{\infty}\right)$ and $S_{2}^{\#}: \mathbb{Q} \mathfrak{C} \rightarrow \mathbb{Q}+$ $\mathcal{M}_{\mathbb{Q},-}^{R}\left(\mathbb{C}^{\infty}\right)$, with $S_{1}^{\#}(\{0\},\{0\})=1, S_{2}^{\#}(\{0\},\{0\})=1$, such that

$$
S^{\#}=\left(S_{1}^{\#}\right)^{*(-1)} * S_{2}^{\#} \text {. }
$$


We shall provide an interpretation of the linear maps $S_{1}^{o}, S_{2}^{o}$ and $S_{1}^{c}, S_{2}^{c}$ in the context of EulerMaclaurin formula. Before that, we deduce a relation between the factors in open and closed cases.

Proposition 4.12. For $\left(C, \Lambda_{C}\right) \in \mathfrak{C}$, we have

$$
S_{2}^{o}\left(C, \Lambda_{C}\right)=S_{2}^{c}\left(C, \Lambda_{C}\right)
$$

and

$$
\left(S_{1}^{c}\right)^{*(-1)}\left(C, \Lambda_{C}\right)=\sum_{G \leq C}\left(S_{1}^{o}\right)^{*(-1)}\left(G, \Lambda_{G}\right)
$$

Proof. Let $\left(C, \Lambda_{C}\right) \in$ C. By Eqs. (37) and (40) we have

$$
\begin{aligned}
S^{c}\left(C, \Lambda_{C}\right) & =\sum_{F \leq C} S^{o}\left(F, \Lambda_{F}\right) \\
& =\sum_{F \leq C} \sum_{G \leq F}\left(S_{1}^{o}\right)^{*(-1)}\left(t(F, G), \Lambda_{t(F, G)}\right) S_{2}^{o}\left(G, \Lambda_{G}\right) \\
& =\sum_{G \leq C}\left(\sum_{G \leq F \leq C}\left(S_{1}^{o}\right)^{*(-1)}\left(t(F, G), \Lambda_{t(F, G)}\right)\right) S_{2}^{o}\left(G, \Lambda_{G}\right) .
\end{aligned}
$$

Let $B\left(C, \Lambda_{C}\right)$ denote the right hand side of Eq. (45). Then we have

$$
B\left(t(C, G), \Lambda_{t(C, G)}\right)=\sum_{H \leq t(C, G)}\left(S_{1}^{0}\right)^{*(-1)}\left(H, \Lambda_{H}\right)=\sum_{G \leq F \leq C}\left(S_{1}^{o}\right)^{*(-1)}\left(t(F, G), \Lambda_{t(F, G)}\right)
$$

by Proposition 2.22. (目). Thus $S^{c}\left(C, \Lambda_{C}\right)=B * S_{2}^{o}$. Since the ranges of $B$ and $S_{2}^{o}$ are in $\mathcal{M}_{\mathbb{Q},+}$ and $\mathcal{M}_{\mathbb{Q},-}$ respectively, the desired equations hold from the uniqueness of the Algebraic Birkhoff Factorization $S^{c}=\left(S_{1}^{c}\right)^{*(-1)} * S_{2}^{c}$.

4.2.3. Continuous subdivision property of $S_{2}^{c}$. Now let us study the subdivision properties of factors in the Algebraic Birkhoff Factorizations for $S^{c}$ and $S^{c}$. Let

$$
\mu^{o}=\left(S_{1}^{o}\right)^{*(-1)}, \quad \mu^{c}=\left(S_{1}^{c}\right)^{*(-1)} .
$$

Theorem 4.13. The linear maps $\mu^{o}$ and $\mu^{c}$ on $\mathbb{Q}$ have the projection formulae:

$$
\mu^{o}=\pi_{+} S^{o} \text { and } \mu^{c}=\pi_{+} S^{c} .
$$

Proof. Let $\left(C, \Lambda_{C}\right)$ be a lattice cone and $\left(F, \Lambda_{F}\right)$ be a face of it. Since the linear spaces lin $F$ and $\operatorname{lin} t(C, F)$ are perpendicular in $V$ for the inner product $Q(\cdot, \cdot)$, the linear functions from $F$ and from $t(C, F)$ are perpendicular. Thus, for $F \neq\{0\}$ we have

$$
\pi_{+}\left(\left(S_{1}^{o}\right)^{*(-1)}\left(t(C, F), \Lambda_{t(C, F)}\right)\left(\mathrm{id}-\pi_{+}\right)\left(S_{2}^{o}\left(F, \Lambda_{F}\right)\right)\right)=0 .
$$

It then follows from Theorem 4.10 that, for $\left(C, \Lambda_{C}\right) \neq(\{0\},\{0\})$

$$
\begin{gathered}
S_{1}^{o}\left(C, \Lambda_{C}\right)=-\pi_{+}\left(S^{o}\left(C, \Lambda_{C}\right)+\sum_{\{0\} \nsupseteq F \nsupseteq C} S_{1}^{o}\left(t(C, F), \Lambda_{t(C, F)}\right) S^{o}\left(F, \Lambda_{F}\right)\right) \\
=-\pi_{+}\left(S^{o}\left(C, \Lambda_{C}\right)+\sum_{\{0\} \nsupseteq F \nsupseteq C} S_{1}^{o}\left(t(C, F), \Lambda_{t(C, F)}\right) \pi_{+}\left(S^{o}\left(F, \Lambda_{F}\right)\right)\right)
\end{gathered}
$$




$$
=-\pi_{+}\left(S^{o}\left(C, \Lambda_{C}\right)\right)-\sum_{\{0\} \nsupseteq F \nsupseteq C} S_{1}^{o}\left(t(C, F), \Lambda_{t(C, F)}\right) \pi_{+}\left(S^{o}\left(F, \Lambda_{F}\right)\right) .
$$

Hence,

$$
-\left(S_{1}^{o} *\left(\pi_{+} S^{o}\right)\right)\left(C, \Lambda_{C}\right)=0 \text { for }\left(C, \Lambda_{C}\right) \neq(\{0\},\{0\}),
$$

which combined with $\left(S_{1}^{o} *\left(\pi_{+} S^{o}\right)\right)(\{0\},\{0\})=1$ yields

$$
\mu^{o}=\left(S_{1}^{o}\right)^{*(-1)}=\pi_{+} S^{o} .
$$

The same proof yields the corresponding formula for the closed case.

We have the following direct consequences.

Corollary 4.14. $\quad$ (a) $\mu^{o}$ has the discrete open subdivision property and $\mu^{c}$ has the discrete closed subdivision property.

(b) $S_{2}^{c}$ and thus $S_{2}^{o}$ have the continuous subdivision property.

Proof. (a) follows from Theorem 4.13 and the linearity of $\pi_{+}$. Then (D) follows from Corollary 4.11 and Theorem 3.13 .

4.3. Euler-Maclaurin formula. With all the preparations accumulated so far, we are ready to derive Euler-Maclaurin formulae for lattice cones. We keep the setup in Section 4.2.3. We identify the map $S_{2}^{c}$ for smooth lattice cones first. Let $\pi_{ \pm}$be the projection of $\mathcal{M}_{\mathbb{Q}}\left(\mathbb{C}^{\infty}\right)$ to $\mathcal{M}_{\mathbb{Q}, \pm}\left(\mathbb{C}^{\infty}\right)$.

Proposition 4.15. For a smooth lattice cone $\left(C, \Lambda_{C}\right)$, we have

$$
\pi_{-} S^{c}\left(C, \Lambda_{C}\right)(\vec{\varepsilon})=\sum_{F \nsupseteq C} \pi_{+} S^{c}\left(t(C, F), \Lambda_{t(C, F)}\right)(\vec{\varepsilon}) I\left(F, \Lambda_{F}\right)(\vec{\varepsilon}) .
$$

Proof. Let $\left(C, \Lambda_{C}\right)$ be a smooth lattice cone with primary generators $v_{1}, \cdots, v_{n} \in \Lambda_{C}$, and let $L_{i}=$ $L_{v_{i}}, i=1, \cdots, n$, be the linear function $\left\langle v_{i}, \vec{\varepsilon}\right\rangle$ where $\vec{\varepsilon} \in V^{*}$. Furthermore, let $\frac{1}{1-e^{x}}=-\frac{1}{x}+h(x)$ be the decomposition of the function $\frac{1}{1-e^{x}}$ into its singular part and holomorphic part. Then

$$
S^{c}\left(C, \Lambda_{C}\right)(\vec{\varepsilon})=\prod_{i=1}^{n} \frac{1}{1-e^{L_{i}}}=\prod_{i=1}^{n}\left(I\left(L_{i}\right)+h\left(L_{i}\right)\right), \quad I\left(L_{i}\right)=-\frac{1}{L_{i}} .
$$

For any nonempty subset $J \subset[n]$, let $L_{J}=\prod_{i \in J} L_{i}$ and, as a convention, let $L_{\emptyset}=1$. then the denominators in the expansion are of the form $L_{J}$ for some nonempty $J \subset[n]$.

Note that any face $\left(F, \Lambda_{F}\right)$ of $\left(C, \Lambda_{C}\right)$ is of the form $\left\langle v_{i} \mid i \in J\right\rangle$ for $\emptyset \neq J \subseteq[n]$. Thus we focus on the polar germ of the form $L_{J} g$ with $g$ a holomorphic function in linear forms perpendicular to $L_{i}, i \in J$. Notice in this case the lattice cone is smooth, thus $I\left(F, \Lambda_{F}\right)(\vec{\varepsilon})=L_{J}$. By our decomposition procedure, terms of this type come from the projection of

$$
L_{J} \prod_{i \in[n]-J} \frac{1}{1-e^{L_{i}}} .
$$

For any vector $v$ and any non-empty subset $K \subset[n]$, let $v^{K}$ be the projection of $v$ to the orthogonal subspace to the subspace spanned by $v_{j}, j \in K$, and $L_{v}^{J}$ be the corresponding linear function. We also use $v^{J K}$ and $L_{v}^{J K}$ to denote the vector $\left(v^{J}\right)^{K}$ and the corresponding linear function. With these notations we have

$$
L_{i}=L_{i}^{K}+\sum_{j \in K} a_{i j} L_{j}
$$


for some constants $a_{i j}, j \in K$.

The part of the form $L_{J} g$ in $\pi_{-} S^{c}\left(C, \Lambda_{C}\right)$ coincides with the corresponding part in

$$
\pi_{-}\left(L_{J} \prod_{i \in[n]-J}\left(I\left(L_{i}\right)+h\left(L_{i}^{J}\right)\right)\right) .
$$

Now let us determine the contribution from

$$
L_{J} L_{K-J} \prod_{i \in[n]-K} h\left(L_{i}^{J}\right),
$$

with $J \subset K \varsubsetneqq[n]$. By above notation, and for $\ell \in[n]-K$, we have

$$
\begin{aligned}
L_{\ell}^{J} & =L_{\ell}^{J K}+\sum_{i \in K} b_{\ell i} L_{i} \\
& =L_{\ell}^{J K}+\sum_{i \in J} b_{\ell i} L_{i}+\sum_{i \in K-J} b_{\ell i} L_{i}^{J}+\sum_{i \in K_{J}} \sum_{j \in J} b_{\ell i} a_{i j} L_{j} \\
& =L_{\ell}^{J K}+\sum_{i \in K-J} b_{\ell i} L_{i}^{J}+\sum_{i \in J} c_{\ell i} L_{i} .
\end{aligned}
$$

Since the spaces spanned by $\left\{v_{i} \mid i \in K\right\}$ and by $\left\{v_{i} \mid i \in J\right\} \cup\left\{v_{j}^{J} \mid j \in K-J\right\}$ coincide, for any vector, its orthogonal projection to the space spanned by $\left\{v_{i} \mid i \in K\right\}$ and to the space spanned by by $\left\{v_{i} \mid i \in J\right\} \cup\left\{v_{j}^{J} \mid j \in K-J\right\}$ are the same.

Therefore,

$$
L_{\ell}^{J}=L_{\ell}^{J K}+\sum_{i \in K-J} b_{\ell i} L_{i}+\sum_{i \in J} b_{\ell i} L_{i}
$$

is the the projections of $L_{\ell}^{J}$ with respect to the spaces spanned by $\left\{v_{i} \mid i \in K\right\}$, and

$$
L_{\ell}^{J}=L_{\ell}^{J K}+\sum_{i \in K-J} b_{\ell i} L_{i}^{J}+\sum_{i \in J} c_{\ell i} L_{i}
$$

is the the projections of $L_{\ell}^{J}$ with respect to the spaces spanned by $\left\{v_{i} \mid i \in J\right\} \cup\left\{v_{j}^{J} \mid j \in K-J\right\}$.

By direct calculations, the polar germs of type $L_{J} g$ arising from $L_{J} \prod_{i \in K-J} I\left(L_{i}\right) \prod_{i \in[n]-K}\left(L_{i}^{J}\right)^{\alpha_{i}}$ coincide with those from $L_{J} \prod_{i \in K-J} I\left(L_{i}^{J}\right) \prod_{i \in[n]-K}\left(L_{i}^{J}\right)^{\alpha_{i}}$, for fixed $a_{i} \in \mathbb{Z}_{\geq 0}, i \in K-J$. Therefore, the polar germs of type $L_{J} g$ arising from $L_{J} \prod_{i \in K-J} I\left(L_{i}\right) \prod_{i \in[n]-K} h\left(L_{i}^{J}\right)$ coincide with those from $L_{J} \prod_{i \in K-J} I\left(L_{i}^{J}\right) \prod_{i \in[n]-K} h\left(L_{i}^{J}\right)$. Consequently, the corresponding terms in $L_{J} \prod_{i \in[n]-J}\left(I\left(L_{i}\right)+h\left(L_{i}^{J}\right)\right)$ coincide with those from $L_{J} \prod_{i \in[n]-J}\left(I\left(L_{i}^{J}+h\left(L_{i}^{J}\right)\right)\right.$, which is of the form $I\left(L_{1}\right) \cdots I\left(L_{m}\right) \pi_{+} S^{c}\left(t(C, F), \Lambda_{t(C, F)}\right)$. This completes the proof.

Corollary 4.16. Let $\left(C, \Lambda_{C}\right)$ be a smooth lattice cone, then

$$
S_{2}^{c}\left(C, \Lambda_{C}\right)(\vec{\varepsilon})=I\left(C, \Lambda_{C}\right)(\vec{\varepsilon})
$$

and we have the Euler-Maclaurin formula:

$$
S^{c}\left(C, \Lambda_{C}\right)=\sum_{F \leq C} \pi_{+} S^{c}\left(t(C, F), \Lambda_{t(C, F)}\right) I\left(F, \Lambda_{F}\right) .
$$


Proof. Adding $\pi_{+} S^{c}\left(C, \Lambda_{C}\right)$ to both sides of the equation in Proposition 4.15, we obtain

$$
S^{c}\left(C, \Lambda_{C}\right)(\vec{\varepsilon})=\sum_{F \leq C} \pi_{+} S^{c}\left(t(C, F), \Lambda_{t(C, F)}\right)(\vec{\varepsilon}) I\left(F, \Lambda_{F}\right)(\vec{\varepsilon})
$$

Then the corollary follows from the uniqueness of the Algebraic Birkhoff Factorization in Corollary 4.11 since $I\left(F, \Lambda_{F}\right)$ is in $\mathcal{M}_{\mathbb{Q},-}\left(\mathbb{C}^{\infty}\right)$.

We are now ready to give the Euler-Maclaurin formula for lattice cones. Recall that a cone $C$ in a lattice vector space $\left(V, \Lambda_{V}\right)$ can be viewed as a lattice cone $\left(C, \operatorname{lin}(C) \cap \Lambda_{V}\right)$. Our approach by means of the Algebraic Birkhoff Factorization applied to $\left(C, \operatorname{lin}(C) \cap \Lambda_{V}\right)$ yields back BerlineVergne's Euler-Maclaurin formulae for the cone $C$ [1] together with a new piece of information, namely that the interpolation function $\mu^{c}$ actually boils down to the holomorphic projection of the exponential sum.

Theorem 4.17. Let $\left(C, \Lambda_{C}\right) \in \mathfrak{C}_{k}$ be a lattice cone and $\vec{\varepsilon} \in V_{k}^{*}$. Then

$$
S_{2}^{c}\left(C, \Lambda_{C}\right)(\vec{\varepsilon})=I\left(C, \Lambda_{C}\right)(\vec{\varepsilon})
$$

(Euler-Maclaurin formula) $\quad S^{c}\left(C, \Lambda_{C}\right)=\sum_{F \leq C} \mu^{c}\left(t(C, F), \Lambda_{t(C, F)}\right) I\left(F, \Lambda_{F}\right)$

and the interpolation function $\mu^{c}$ coincides with the holomorphic projection of the discrete sum,

$$
\mu^{c}=\pi_{+} S^{c} .
$$

Proof. Proposition 4.15 shows that $S_{2}^{c}$ agrees with $I$ for smooth lattice cones. By Corollary 4.14.(何), $S_{2}^{c}$ has continuous subdivision property, which is known to hold for $I$ also. Therefore, by taking smooth subdivisions, they agree for all lattice cones, proving the first equation. Then the second and third equations follow from Corollary 4.11 and Proposition 4.13 respectively.

In view of Proposition 4.12, the Algebraic Birkhoff Factorization for $S^{o}$ yields an open variant of the Euler-Maclaurin formula by applying the factorization to the linear map $S^{o}: \mathbb{Q C} \rightarrow$ $\mathcal{M}_{\mathbb{Q}}\left(\mathbb{C}^{\infty}\right)$.

Corollary 4.18. (Open Euler-Maclaurin formula) Let $\left(C, \Lambda_{C}\right) \in \mathfrak{C}_{k}$ be a lattice cone and $\vec{\varepsilon} \in V_{k}^{*}$. Then

$$
S_{2}^{o}\left(C, \Lambda_{C}\right)(\vec{\varepsilon})=I\left(C, \Lambda_{C}\right)(\vec{\varepsilon}),
$$

(Euler-Maclaurin formula) $\quad S^{o}\left(C, \Lambda_{C}\right)=\sum_{F \leq C} \mu^{o}\left(t(C, F), \Lambda_{t(C, F)}\right) I\left(F, \Lambda_{F}\right)$

and we have a projection formula for the interpolate function $\mu^{\circ}$,

$$
\mu^{o}=\pi_{+} S^{o} \text {. }
$$

Acknowledgements: This work is supported by the National Natural Science Foundation of China (Grant No. 11071176, 11221101 and 11371178) and the National Science Foundation of US (Grant No. DMS 1001855). The authors thank Kavli Institute for Theoretical Physics China (KITPC) and Morningside Center of Mathematics (MCM) in Beijing where part of the work was carried out. The second author thanks Sichuan University, Lanzhou University and Capital Normal University for their kind hospitality. 


\section{REFERENCES}

[1] N. Berline and M. Vergne, Local Euler-Maclaurin formula for polytopes, Mosc. Math. J. 7 (2007) 355386. 2, 目, 6, 耳, 22, 30

[2] M. Brion and M. Vergne, Lattice points in simple polytopes, J. Amer. Math. Soc. 10 (1997), 371-392. 1]

[3] J.-L. Brylinsky and B. Zhang, Equivariant Todd classes for Toric varieties, arXiv:math/0311318

[4] S. E. Cappell and J. L. Shaneson, Genera of algebraic varieties and counting of lattice points, Bull. Amer. Math. Soc. 30 (1994), 62-69. 11

[5] A. Connes and D. Kreimer, Hopf algebras, Renormalization and Noncommutative Geometry, Comm. Math. Phys. 199 (1988) 203-242.2, 25

[6] W. Fulton: Introduction to Toric Varieties, Princeton University Press, 1993. ., 13

[7] S. Garoufalidis and J. Pommersheim, Sum-integral interpolators and the Euler-Maclaurin formula for polytopes, Trans. Amer. Math. Soc. 364 (2012) 2933-2958. 6, 22

[8] V. Guillemin and S. Sternberg, Riemann sums over polytopes, Annales de l'institut Fourier 57 (2007), 2183-2195. 11

[9] L. Guo, An Introduction to Rota-Baxter Algebra, International Press, 2012.9

[10] L. Guo, S. Paycha and B. Zhang, Conical zeta values and their double subdivision relations, Adv. Math. 252 (2014) 343-381. 月, 22, 24

[11] L. Guo, S. Paycha and B. Zhang, Residue of meromorphic functions with linear poles, arXiv:1501.00426v1. 3 , 22, 26

[12] G. Hardy, Divergent Series, Oxford University Press, 1967. 1]

[13] J. Lawrence, Rational-function-valued valuations on polyhedra, DIMACS Ser. Discrete Math. Theoret. Comput. Sci. 6 (1991) 199-208. 22

[14] V.I. Lomonosov (originator), Positive cone, Encyclopedia of Mathematics. URL: http://www.encyclopediaofmath.org/index.php?title=Positive_cone\&oldid=15148. 5

[15] J.-L. Loday and B. Vallette, Algebraic Operads, Grundlehren Math. Wiss. 346, Springer, Heidelberg, 2012. 10

[16] D. Manchon, Hopf algebras, from basics to applications to renormalization, Comptes-rendus des Rencontres mathématiques de Glanon 2001 (2003); Hopf algebras in renormalization, Handbook of algebra, Vol. 5 (M. Hazewinkel ed.) (2008). 9,25

[17] A. Khovanskii and A. Pukhlikov, A RiemannRoch theorem for integrals and sums of quasipolynomials over virtual polytopes, Algebra and Analysis, v.4, N4, 188-216, 1992; translation in St. Petersburg Math. J. 4 (1993), 789812. 1

[18] G. Ziegler, Lectures on Polytopes, Graduate Texts in Mathematics, Springer Verlag, 2nd ed. 1994. 4

Department of Mathematics and Computer Science, Rutgers University, Newark, NJ 07102, USA

E-mail address: liguo@rutgers.edu

Institute of Mathematics, University of Potsdam, Am Neuen Palais 10, D-14469 Potsdam, Germany

E-mail address: paycha@math.uni-potsdam.de

School of Mathematics, Yangtze Center of Mathematics, Sichuan University, Chengdu, 610064, P. R. China

E-mail address: zhangbin@scu.edu.cn 